This is a postprint/Accepted version of the following published document:

Huete, C., et. al. Weak-shock interactions with transonic laminar mixing layers of fuels for high-speed propulsion. In: AIAA journal, 54(3), March 2016, Pp. 962-975

DOI: https://doi.org/10.2514/1.J054419

(C) 2015 by the authors. Published by the American Institute of Aeronautics and Astronautics, Inc., with permission. 


\title{
Weak-Shock Interactions with Transonic Laminar Mixing Layers of Fuels for High-Speed Propulsion
}

\author{
César Huete $^{*}$, Javier Urzay ${ }^{\dagger}$, Antonio L. Sánchez ${ }^{\ddagger}$, Forman A. Williams ${ }^{\S}$ \\ * $₫$ University of California San Diego, La Jolla, CA 92093-0411 \\ ${ }^{\dagger}$ Center for Turbulence Research, Stanford University, Stanford CA 94305-3024
}

This article extends to transonic mixing layers an analysis of Lighthill on the interaction between weak shocks and laminar boundary layers. As in the previous work, the analysis is carried out under linear-inviscid assumptions for the perturbation field, with streamwise changes of the base flow neglected, as is appropriate given the slenderness of the mixing-layer flow. The steady-disturbance profile is determined by taking a Fourier transform along the longitudinal coordinate. Closed-form analytical functions for the pressure field are derived in the small- and large-wavenumber limits, and vorticity disturbances are obtained as functions of the pressure perturbations. The analysis is particularized to ethylene-air and hydrogen-air mixing layers, whose dynamics are of current interest for hypersonic propulsion. The results provide, in particular, the effective distance of upstream influence of the pressure perturbation in the subsonic stream. The resulting value, which scales with the thickness of the subsonic layer, is much smaller than the upstream influence distances encountered in boundary layers. This study may serve as a basis to understand shock-induced autoignition and flame-holding phenomena in simplified versions of non-premixed supersonic-combustion problems.

\section{Nomenclature}

$\begin{array}{llll}C_{p} & \text { Normalized specific heat } & g_{1}, G_{1} & \text { Physical- and Fourier-space reflected } \\ D & \text { Normalized binary diffusion coefficient } & & \text { pressure perturbation } \\ f_{1}, F_{1} & \begin{array}{l}\text { Physical- and Fourier-space incident } \\ \quad\end{array} & J & \text { Normalized fuel-diffusion flux } \\ & \text { pressure perturbation } & k & \text { Normalized streamwise wavenumber }\end{array}$

\footnotetext{
* Postdoctoral Researcher, Department of Mechanical and Aerospace Engineering, University of California San Diego, chuete@ucsd. edu

${ }^{\dagger}$ Engineering Research Associate, Center for Turbulence Research, Stanford University.

${ }^{\ddagger}$ Professor, Department of Mechanical and Aerospace Engineering, University of California San Diego

$\S$ Professor, Department of Mechanical and Aerospace Engineering, University of California San Diego. AIAA Fellow.
} 


\begin{tabular}{|c|c|c|c|}
\hline$L e$ & Fuel Lewis number & $\alpha$ & Thermal-diffusion factor \\
\hline$M$ & Mach number & $\beta$ & Cotangent of Mach angle \\
\hline \multirow[t]{2}{*}{$p, \mathcal{P}$} & Physical- and Fourier-space & $\gamma$ & Ratio of specific heats \\
\hline & pressure perturbations & $\epsilon$ & Normalized perturbation amplitude \\
\hline $\operatorname{Pr}$ & Prandtl number & $\eta$ & Selfsimilar variable \\
\hline$R$ & Normalized density & $\Theta, \theta$ & Physical- and Fourier-space temperature \\
\hline$R e$ & Reynolds number & & perturbations \\
\hline \multirow[t]{2}{*}{$L, \ell_{m}$} & Mixing-layer development distance & $\mu$ & Normalized mean viscosity \\
\hline & and thickness & $\omega, \varpi$ & Physical- and Fourier-space \\
\hline$T$ & Normalized temperature & & vorticity perturbations \\
\hline$U, V$ & Normalized streamwise & $\Omega$ & Vorticity production factor \\
\hline & and transverse velocities & & \\
\hline \multirow[t]{2}{*}{$u, v$} & Normalized streamwise and transverse & A. S & scripts \\
\hline & velocity perturbations & 1 & Supersonic air stream \\
\hline$W$ & Normalized mean molecular weight & 2 & Subsonic fuel stream \\
\hline$Y, y, \mathcal{Y}$ & $\begin{array}{l}\text { Base-flow, perturbation, and Fourier- } \\
\text { transformed fuel mass fractions }\end{array}$ & & \\
\hline$X, Z$ & Streamwise and transverse global coordinates & B. $\mathrm{S}$ & rscripts \\
\hline$x, z$ & Normalized streamwise and transverse & ' & Dimensional variables \\
\hline
\end{tabular}

\section{Introduction}

Mixing layers and shock waves are two different phenomena that coexist in hypersonic and supersonic propulsion devices. For instance, in supersonic-combustion ramjets (SCRAMJETS), shock waves are typically generated ahead of the combustion zone, where the supersonic incoming flow enters a converging nozzle and interacts with wedged walls and fuel injectors. Along its path through the combustor, the flow is subject to complex shock trains and expansion waves [1].

In SCRAMJETS, shock waves can interact with the flow in many different ways. For instance, shocks may disturb 
the flow near the walls leading to sudden transition to turbulence and augmented wall heating in boundary layers. The corresponding shock/boundary-layer interaction problem is one of high practical relevance that has received a large amount of attention in recent years [2, 3, 4, 5]. A relatively less known interaction occurs when shocks impinge on chemically-reacting mixing layers of fuel and oxidizer. To illustrate the relevance of shock/mixing-layer interaction phenomena, consider the following standard fuel-injection configurations employed in SCRAMJETS. In one configuration, the shock waves interact with the mixing layer that separates the supersonic incoming hot-air stream and the subsonic fuel flow, and which is generated downstream from a strut fuel injector (see Figs. 5 and 11 in Ref. [6]). Similarly, in configurations with jet-in-crossflow fuel injection, a reflected bow shock interacts with the mixing layer generated from the aerodynamics of the fuel jet as it flows into the supersonic incoming hot-air stream (see, for instance, Fig. 4 in Ref. [7]). In all cases, since the residence time of the reactants in the combustor is short in supersonic regimes, ignition typically cannot be achieved by relying on diffusion and heat conduction alone. Shock waves may help, however, to heat the mixture and speed-up the mixing process, the former arising from the inherent temperature rise across the shock wave, and the latter associated with the interaction of the shock with the non-uniform flow $[5,6,7,8,9,10,11,12,13,14,15,16,17,18]$

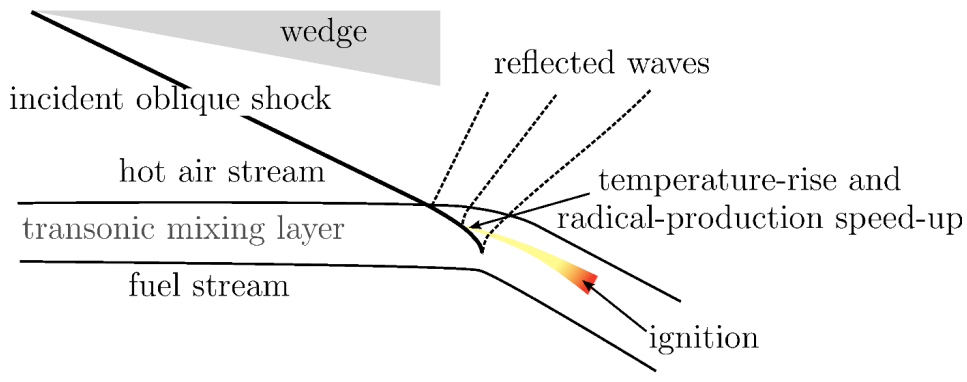

Figure 1. Sketch of the general configuration.

Although the aerodynamic interactions described above are predominantly encountered in highly turbulent flows in practical applications, analytical solutions to related simplified laminar problems can be advantageous in studying such supersonic-combustion processes, helping to clarify the real configuration, not only for increasing understanding but also for suggesting scaling concepts that may prove useful in formulating subgrid-scale models. The present work, which is of that type, pertains to transonic laminar mixing layers formed by fuels employed in supersonic combustion and subjected to impingement by a shock from the air stream, roughly as illustrated in Fig. 1. As a first step, an inert mixing layer is considered, and the shocks are assumed to be sufficiently weak to be treated as linear perturbations of 
the base flow, the non-linear influences of finite-amplitude shocks on combustion and the effects of heat release being deferred to later investigations. A crucial asset for the present investigation is the earlier work by Lighthill [19, 20], which was instrumental in understanding the fundamental dynamics of weak-shock impingement on wall boundary layers. Initially presented in a physical context, it was later shown by Stewartson and Williams [21] that this type of problem, involving linear (weak-shock) perturbations of a laminar viscous region at large values of the Reynolds number, $R e$, can be treated rigorously through matched asymptotic expansions for $R e$ approaching infinity, resulting in a triple-deck theory. This has been explained carefully in more recent reviews, such as that of Nayfeh [22], where the relationships to other triple-deck problems are made clear. The mixing-layer problem to be addressed here turns out to be a particularly simple version of multi-scale problems of this type, for example because it is unnecessary to deal with the bottom (low-speed, viscous, incompressible) deck.

The objective of this study is to describe, by using asymptotic analysis, the effect of a weak shock on an inert laminar transonic mixing layer. Particular attention is given to the effect of the perturbations in the nonslender interaction region found around the impingement point, giving a problem that can be treated using Lighthill's theory on shock/boundary-layer interaction [19, 20]. Molecular transport effects, which determine the slow evolution of the mixing layer flow upstream from the impingement location, have, however, a negligible effect on the perturbations in the interaction region, because the local Reynolds number there is large. Correspondingly, since the streamwise extent of the interaction region, of the order of the mixing-layer thickness, is much smaller than the mixing-layer development length, the streamwise variations of the background flow variables can be neglected when writing the linearized problem for the perturbations induced by the weak shock. Therefore, for the base flow only transverse changes in the density and velocity are considered, while the background pressure field is assumed to be constant along and across the mixing layer in the first approximation. These approximations engender analytic solutions.

The paper is structured as follows: The background laminar mixing layer and the asymptotic perturbation theory are formulated in Section II. The perturbation pressure field is analyzed by means of a Fourier transformation along the streamwise direction. An ordinary differential equation for the pressure perturbations, as functions of the transverse variable, is obtained. The asymptotic results for high-frequency and low-frequency disturbances as functions of the transverse coordinate and the frequency are provided in Section III, followed in Section IV by an analysis of the upstream decay of the disturbance. Although the general analysis will be performed for a weak pressure perturbation of arbitrary shape, the specific interaction with a weak step-pressure wave, emulating the weak shock, is addressed in 
Section V, where the pressure-perturbation distribution throughout the mixing layer is computed and analyzed. The effects that the weak shock causes on the vorticity field are analyzed separately in Section VI. Finally, the conclusions are summarized in Section VII.

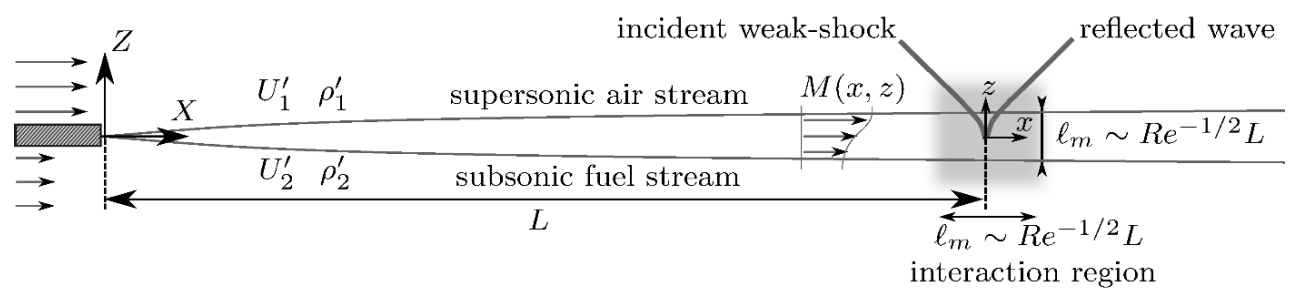

Figure 2. Sketch of the model problem.

It is relevant to point out that there are three previous investigations of interactions of oblique shocks with mixing regions, although the specific transonic problems addressed here have not been treated. Riley [23] employed the same methods adopted here to analyze the interaction of a shock from a supersonic stream, incident at a fixed plane on a shear layer of infinite extent, in which the Mach number approaches zero at infinity. Although he did not consider the mixing-layer composition and temperature profiles, such as those that we analyze, instead studying influences of two model transverse distributions of the Mach number, a number of the results of his theory are the same as ours. Moeckel [24] derived a simplified method for describing shock shapes in purely supersonic mixing regions. The same method was employed later by Buttsworth [25], who attempted computations of vorticity fields in mixing layers of ideal gases with similar and different thermodynamic properties, his investigation being motivated by the same supersonic-combustion applications that led to our study. All of these excellent contributions explain methods, not investigated here, for taking into account influences of finite amplitudes of the incident waves.

\section{Problem formulation}

We consider the interaction of a steady transonic mixing layer -separating a supersonic air stream from a subsonic fuel stream- with a small, external, steady, pressure perturbation approaching from the supersonic side. The mixing layer develops downstream from a separating splitter plate, with the perturbation reaching the mixing layer at a downstream distance $X=L$, as indicated in Fig. 2. The distribution of flow properties across the laminar mixing layer depends on the type of air-fuel mixture, with two different relevant cases considered below. A first set of 
integrations neglects variations of the mean molecular weight and assumes a unity Lewis number when describing the fuel diffusion velocity, with thermal diffusion neglected. This simplified case is representative of fuels that have properties close to those of air, such as ethylene, which has been employed in recent supersonic combustion research [26]. Properties of ethylene [27] are not exactly those of air, but, as will be shown, they are sufficiently close for its behavior to be approximated well by that of the simplified case. Investigation of hydrogen, also a promising candidate for high-speed combustion because of its favorable chemical properties (high mass-based energy density and high reactivity), requires a separate analysis including consideration of its specific physical properties, i.e., low molecular weight, high diffusivity, and non-negligible thermal diffusion.

The relevant Reynolds number of the flow $R e=\rho_{1}^{\prime} U_{1}^{\prime} L / \mu_{1}^{\prime}$, based on the velocity $U_{1}^{\prime}$, density $\rho_{1}^{\prime}$, and shear viscosity $\mu_{1}^{\prime}$ of the supersonic stream is assumed to be moderately large and comparable in magnitude to the corresponding value $\rho_{2}^{\prime} U_{2} L / \mu_{2}^{\prime}$ based on the subsonic-stream properties. This results in a slender mixing layer, whose characteristic thickness increases according to $\left[\left(\mu_{1}^{\prime} / \rho_{1}^{\prime}\right) X / U_{1}\right]^{1 / 2}$, reaching a value $\ell_{m}$ of order $R e^{-1 / 2} L \ll L$ at $X=L$. Since the relative magnitude of the external pressure perturbation $\epsilon$ is assumed to be infinitesimally small, in the first approximation the flow variables are given by those of the unperturbed laminar mixing layer, which is known to possess a selfsimilar solution, to be described below in Section III.A. The interaction of the perturbation with the mixing layer occurs in a nonslender region of characteristic size $\ell_{m}$, where the relevant local Reynolds number is $\rho_{1}^{\prime} U_{1}^{\prime} \ell_{m} / \mu_{1}^{\prime} \sim R e^{1 / 2} \gg 1$. As a result, in the double limit $R e \gg 1$ and $\epsilon \ll 1$ the interaction region can be analyzed, following Lighthill's seminal work [19, 20], by linearizing the conservation equations around the background solution, with molecular-transport terms neglected at leading order, along with the small streamwise variations of the base flow, of order $R e^{-1 / 2}$ for the slender mixing layer considered here.

\section{A. The transonic mixing layer}

In the absence of external perturbations, the transonic mixing layer that develops downstream from the splitter plate possesses a selfsimilar solution in terms of the rescaled transverse coordinate $\eta=Z /\left[\left(\mu_{1}^{\prime} / \rho_{1}^{\prime}\right) X / U_{1}^{\prime}\right]^{1 / 2}$. In the description, the longitudinal and transverse velocity components are scaled with their characteristic values $U_{1}^{\prime}$ and $\left[\left(\mu_{1}^{\prime} / \rho_{1}^{\prime}\right) U_{1}^{\prime} / X\right]^{1 / 2}$ to define the nondimensional functions $U(\eta)$ and $V(\eta)$, while the temperature and density are scaled with their air-side values $T_{1}^{\prime}$ and $\rho_{1}^{\prime}$, respectively, to define $T(\eta)$ and $R(\eta)$. The adiabatic pressure disturbances in the interaction region will be found below to be governed by an equation that depends only on the distribution of Mach number $M(\eta)$ across the mixing layer. Since the ratio $\gamma$ of specific heats is essentially constant in these ideal-gas 
mixtures, the sound speed is inversely proportional to the square root of the density because the pressure does not vary appreciably across the mixing layer. As a result, the distribution of Mach number $M(\eta)$ can be evaluated from the nondimensional velocity and density profiles according to

$$
M(\eta)=M_{1} U(\eta) R(\eta)^{1 / 2}
$$

where $M_{1}>1$ is the Mach number of the air stream, yielding the relation $M_{2}=M_{1} U_{2} R_{2}^{1 / 2}<1$ for the fuel-stream Mach number.

Since nitrogen and oxygen are very similar, they will be treated below as a single species, thereby reducing the mixing process to that of a binary mixture, with the local composition characterized in terms of the fuel mass fraction $Y$. The corresponding fuel diffusion flux, nondimensionalized with $\left[\rho_{1}^{\prime} \mu_{1}^{\prime} U_{1}^{\prime} / X\right]^{1 / 2}$, can be shown to be expressible in the explicit form [28]

$$
J=-\frac{R D}{\operatorname{Pr} L e}\left(\frac{\mathrm{d} Y}{\mathrm{~d} \eta}+\alpha \frac{Y(1-Y)}{T} \frac{\mathrm{d} T}{\mathrm{~d} \eta}\right)
$$

accounting for both species gradient diffusion and thermal diffusion. The latter, the Ludwig-Soret effect, exerts significant influences in laminar hydrogen-air mixing layers, while its reciprocal Onsager property, the Dufour effect, has little influence on the results. Here $\operatorname{Pr}=\mu^{\prime} c_{p} / \lambda$ is the Prandtl number of the gas mixture, assumed here to be constant and equal to $\operatorname{Pr}=0.7$, with $\lambda$ and $c_{p}$ representing the thermal conductivity and the specific heat at constant pressure of the mixture. The ratio of the thermal diffusivity $\lambda /\left(\rho^{\prime} c_{p}\right)$ to the fuel-air binary diffusion coefficient $D^{\prime}$ is the Lewis number, a quantity that depends on the mixture composition through the variation of $\lambda /\left(\rho^{\prime} c_{p}\right)$ with molecular weight. Its value in the air stream $L e=\lambda_{1} /\left(\rho_{1}^{\prime} c_{p 1} D_{1}^{\prime}\right)$ appears multiplying the Prandtl number in (2), which includes the dimensionless binary diffusion coefficient $D=D^{\prime} / D_{1}^{\prime}$, a function of the temperature given below in (8). The thermal diffusion factor $\alpha$ (the ratio of the thermal diffusion coefficient to the product $\left.Y(1-Y) \rho^{\prime} D^{\prime}\right)$ will be taken to be constant, a sufficiently accurate approximation for hydrogen-air mixtures, for which $\alpha \simeq-0.3$ [28].

In terms of the above dimensionless variables, the conservation equations can be written in the boundary-layer 
form

$$
\begin{aligned}
& -\frac{\eta}{2} \frac{\mathrm{d}}{\mathrm{d} \eta}(R U)+\frac{\mathrm{d}}{\mathrm{d} \eta}(R V)=0 \\
& R\left(V-\frac{\eta}{2} U\right) \frac{\mathrm{d} U}{\mathrm{~d} \eta}=\frac{\mathrm{d}}{\mathrm{d} \eta}\left(\mu \frac{\mathrm{d} U}{\mathrm{~d} \eta}\right) \\
& R C_{p}\left(V-\frac{\eta}{2} U\right) \frac{\mathrm{d} T}{\mathrm{~d} \eta}=\frac{\mathrm{d}}{\mathrm{d} \eta}\left(\frac{\mu C_{p}}{P r} \frac{\mathrm{d} T}{\mathrm{~d} \eta}\right)-\left(W_{2}^{-1}-1\right) J \frac{\mathrm{d} T}{\mathrm{~d} \eta} \\
& \quad-\frac{\alpha(\gamma-1)}{W_{2} \gamma} \frac{\mathrm{d}}{\mathrm{d} \eta}(W J T)+(\gamma-1) M_{1}^{2} \mu\left(\frac{\mathrm{d} U}{\mathrm{~d} \eta}\right)^{2}, \\
& R\left(V-\frac{\eta}{2} U\right) \frac{\mathrm{d} Y}{\mathrm{~d} \eta}=-\frac{\mathrm{d} J}{\mathrm{~d} \eta}
\end{aligned}
$$

where $\mu=\mu^{\prime} / \mu_{1}^{\prime}, C_{p}=c_{p} / c_{p 1}$ and $W=W^{\prime} / W_{1}^{\prime}$ (W' denoting molecular weights) are the shear viscosity, specific heat, and mean molecular weight scaled with their air-stream values, and $W_{2}=W_{2}^{\prime} / W_{1}^{\prime}$.

The above equations must be supplemented with the equation of state

$$
R T=W=\frac{1}{1+\left(W_{2}^{-1}-1\right) Y}
$$

and with the expressions

$$
\begin{gathered}
\mu=\frac{1+\left[\left(\mu_{2}^{\prime} / \mu_{1}^{\prime}\right) W_{2}^{-1 / 2}-1\right] Y}{1+\left(W_{2}^{-1 / 2}-1\right) Y} T^{\sigma_{1}}, \\
D=T^{1+\sigma_{1}}, \text { and } C_{p}=\left[1+\left(W_{2}^{-1}-1\right) Y\right] T^{\sigma_{2}},
\end{gathered}
$$

for the variation with temperature and composition of the transport coefficients and specific heat. The representative values $\sigma_{1}=0.7$ and $\sigma_{2}=0.2$ are used below for the temperature exponents. The semi-empiric expression used for the viscosity of a binary mixture, taken from Ref. [29], and that employed for $C_{p}$, which follows from assuming that the molar specific heat at constant pressure is identical for the fuel and the air, are approximate descriptions that give excellent accuracy in many configurations of interest, notably for hydrogen-air mixtures. The temperature variations in (8) and the assumption that the Prandtl number $\operatorname{Pr}=\mu^{\prime} c_{p} / \lambda$ is constant are consistent with a Lewis number $\lambda /\left(\rho^{\prime} c_{p} D^{\prime}\right)$ that has a negligible temperature dependence and a thermal conductivity that increases with temperature according to $\lambda \propto T^{\sigma_{1}+\sigma_{2}}$. In the simplified case that approximates ethylene as the fuel, the dependences on $Y$ disappear from (7) and (8), with $W=W_{2}=1$ and $\mu_{2}^{\prime}=\mu_{1}^{\prime}$, giving $\mu=1, C_{p}=1$, and, in (2), $J=T^{\sigma_{1}}(\mathrm{~d} Y / \mathrm{d} \eta) / P r$.

The problem reduces to the integration of (3)-(6) supplemented with (7) and (8) and subject to the boundary conditions $U=1, T=1$, and $Y=0$ as $\eta \rightarrow \infty$ and $U=U_{2}, T=T_{2}$, and $Y=1$ as $\eta \rightarrow-\infty$, together with the additional boundary condition $M=M_{1} U R^{1 / 2}=1$ at $\eta=0$, stating that the arbitrary origin of the transverse coordinate $\eta$ is selected to be the sonic point. The resulting description is similar to that presented in a previous analysis 

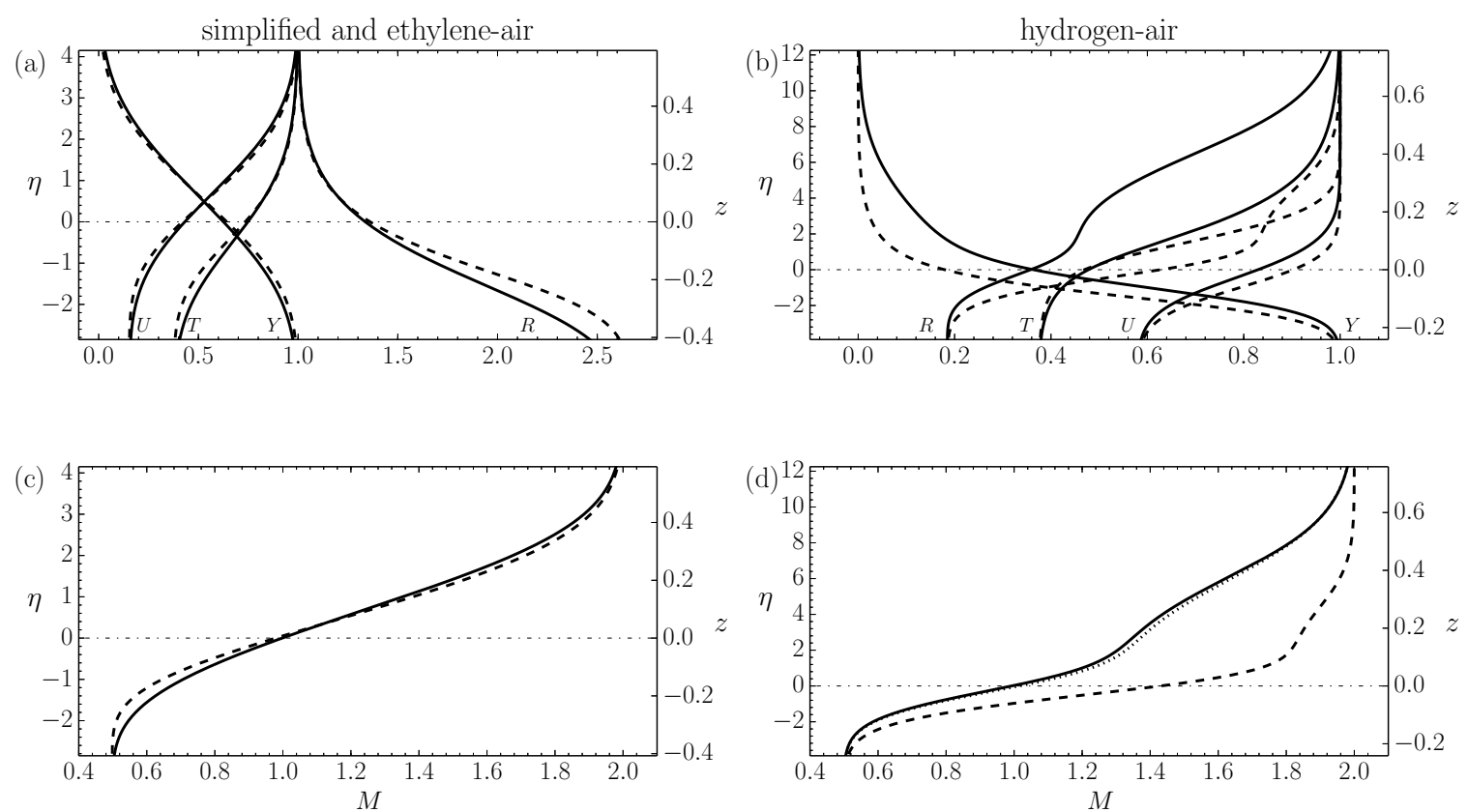

Figure 3. Profiles of $U, T, R, Y$, and $M$ determined by integration of (3)-(6) for simplified and ethylene-air mixing layers (left-hand-side plots) and for a hydrogen-air mixing layer (right-hand-side plots) with $T_{2}=0.375, M_{1}=2$ and $M_{2}=0.5$. In panels (a) and (c), the solid and dashed curves represent the simplified and ethylene-air cases, respectively; in panels (b) and (d), the dashed and dotted curves represent results obtained by removing the Soret and Dufour effects, respectively.

of transient hydrogen-air mixing [30]. A distinguishing feature of the present analysis is the inclusion of the last two terms in (5), which represent, respectively, the Dufour effect, by which an energy flux is generated by gradients of species concentrations, and the heating by viscous dissipation, relevant for the transonic conditions examined here. Integrations were performed for fuel streams composed of hydrogen $\left(L e=0.3, \alpha=-0.3, W_{2}=0.069\right.$, and $\left.\mu_{2}^{\prime}=0.514 \mu_{1}^{\prime}\right)$ and ethylene $\left(L e=1.2, W_{2}=0.97\right.$, and $\mu_{2}^{\prime}=0.6 \mu_{1}^{\prime}$, [27]). Additionally, comparisons were made between ethylene-air and air-air mixing layers (with $L e=1, \alpha=0, W_{2}=1$, and $\mu_{2}^{\prime}=\mu_{1}^{\prime}$ employed for air), which show that both configurations yield qualitatively similar results. Sample profiles of $U, T, R$, and $Y$ obtained for the case $T_{2}=0.375$ with $M_{1}=2$ and $M_{2}=0.5$ are shown in Fig. 3, which also displays the corresponding distributions of Mach number, evaluated from (1).

The results shown in Figs. 3(a) and 3(c) appear quite symmetric, while those for hydrogen-air systems are more irregular, exhibiting, for example, three inflection points in the profiles of density and Mach number. These differences are due to the specific properties of hydrogen, notably its low molecular weight and high diffusivity. Thus, for both the ethylene-air and simplified cases, in which the molecular-weight variation is unimportant, the density decreases as 
the temperature increases across the mixing layer from the subsonic to the supersonic stream, i.e., such that $\mathrm{d} R / \mathrm{d} z<$ 0 everywhere. For the hydrogen-air mixing layer, however, the density changes associated with molecular weight variations are sufficiently large that, for the air-to-fuel temperature ratio selected in the figure, the corresponding density profile becomes an increasing function of $z$. These differences in transverse density gradient will be seen later to have a significant effect on the vorticity generated by baroclinic torque.

The larger thickness of the hydrogen-air mixing layer, seen from the different scales in the left and right columns of Fig. 3(b-d), is a direct consequence of the higher molecular diffusivity of the $\mathrm{H}_{2}$ molecules, apparent in their small Lewis number $L e=0.3$, and of the augmented transport rate associated with thermal diffusion. The latter phenomenon is seen to have a prominent effect on the solution, as was verified by performing calculations with $\alpha=0$, giving the profiles represented by the dashed curves in Fig. 3(b). The modifications in the hydrogen distribution are seen to alter significantly the profiles of the other flow variables, especially those of density and Mach number. In particular, as can be inferred from the resulting $M(\eta)$, in the absence of thermal diffusion the supersonic boundary of the mixing layer would be much closer to the sonic plane. Also of interest is that the Dufour effect comparatively has a much smaller influence on the solution, as revealed by the dotted Mach-number profile in Fig. 3(d), obtained by selectively removing the Dufour term in the energy equation (i.e., setting $\alpha=0$ in (5)). Thermal diffusion therefore is important only through the Soret effect in this problem, as is often the case.

The sonic conditions, denoted by the superscript $*$, are reached at $\eta=0$. At this point, the nondimensional temperature and fuel mass fraction are $T^{*}=0.71$ and $Y^{*}=0.65$ for the simplified case and $T^{*}=0.48$ and $Y^{*}=0.36$ for hydrogen-air mixing layers. Since the shock penetration will be seen to terminate at the sonic point, it is thus found to end closer to the properties of the fuel stream in the simplified case than in the hydrogen-air layer. Largely because of the high sound speed of hydrogen, however, the resulting distance from the sonic point to the subsonic boundary, measured relative to the total mixing-layer thickness, turns out to be considerably smaller in hydrogen-air mixing layers when the Mach numbers of the streams are fixed. This specific characteristic of the mixing layer structure will be seen to have an effect on the rate of decay of the acoustic disturbances. With regard to the fuel-mass-fraction profiles, it is also worth noting that the stoichiometric value is always a very small quantity (e.g. $Y_{s t}=0.063$ and $Y_{s t}=0.028$ for ethylene-air and hydrogen-air mixtures, respectively), so that the most favorable mixing conditions for ignition are always found near the air supersonic stream.

The selfsimilar profiles can be used to evaluate the thickness of the mixing layer. Different definitions are appro- 
priate for different applications. Since the interactions investigated below depend fundamentally on the Mach-number distribution, it seems appropriate to use the condition of achievement of $99 \%$ of the free-stream Mach number as the defining criterion for the location of the upper and lower edges of the mixing layer $\eta_{1}$ and $\eta_{2}$, giving for instance $\eta_{1}=4.15$ and $\eta_{2}=-2.85$ for the ethylene-air case and $\eta_{1}=12.25$ and $\eta_{2}=-3.9$ for the hydrogen-air mixing layer shown in Fig. 3. Correspondingly, the analysis yields the value

$$
\ell_{m}=\left(\eta_{1}-\eta_{2}\right)\left[\left(\mu_{1}^{\prime} / \rho_{1}^{\prime}\right) L / U_{1}^{\prime}\right]^{1 / 2}
$$

for the mixing-layer thickness at $X=L$, to be used below as a scale for the interaction region. Because of the initial factor in this equation, for the conditions of Fig. 3 the hydrogen-air mixing layer is more than twice as thick as the ethylene-air layer. The convective Mach number, although most commonly employed for turbulent mixing layers, is also known to be readily definable for laminar mixing layers [31], namely

$$
M_{c}=\frac{U_{1}^{\prime}-U_{2}^{\prime}}{\left[(\gamma-1) c_{p_{1}} T_{1}^{\prime}\right]^{1 / 2}+\left[(\gamma-1) c_{p_{2}} T_{2}^{\prime}\right]^{1 / 2}}=M_{1} \frac{1-U_{2}}{1+R_{2}^{-1 / 2}},
$$

which yields 1.05 for the ethylene-air case but only 0.25 for hydrogen-air. This trend is similar to the general one found in turbulent mixing layers in that mixing-layer thicknesses decrease with increasing convective Mach numbers [32].

\section{B. The perturbed pressure field}

The interactions of the external pressure perturbation with the mixing layer will be studied in a reference frame whose origin is placed at the intersection of the incident wave with the sonic line, located at $(X, Z)=\left(L, Z^{*}\right)$. Using $\ell_{m}$ as characteristic length results in the local coordinates $x=(X-L) / \ell_{m}$ and $z=\left(Z-Z^{*}\right) / \ell_{m}$. In the interaction region, the streamwise variations of the velocity, density, temperature, and fuel mass fraction of the unperturbed base flow are small, of order $R e^{-1 / 2}$, and they can be therefore neglected in the first approximation, along with the departures of the base-flow pressure from the ambient value $p_{o}^{\prime}$, of order $R e^{-1}$. The external pressure perturbation introduced is assumed to be of order $\epsilon p_{o}^{\prime}$, leading to relative departures from the base flow of order $\epsilon$ given by

$$
\begin{aligned}
& \frac{u^{\prime}}{U_{1}^{\prime}}=U(z)+\epsilon u(x, z), \quad \frac{v^{\prime}}{U_{1}^{\prime}}=R e^{-1 / 2} V(z)+\epsilon v(x, z), \\
& \frac{\rho^{\prime}}{\rho_{1}^{\prime}}=R(z)+\epsilon \rho(x, z), \quad \frac{p^{\prime}-p_{o}^{\prime}}{\gamma p_{o}^{\prime}}=\epsilon p(x, z),
\end{aligned}
$$


where the base profiles $U(z), V(z), R(z)$ can be evaluated from the selfsimilar profiles $U(\eta), V(\eta), R(\eta)$ with use made of $z=\eta /\left(\eta_{1}-\eta_{2}\right)$. The normalized transverse coordinate $z$ is included for completeness in the plots of Fig. 3(ad). Note that, with the scaling introduced, the edges of the mixing layer $z_{1}=\eta_{1} /\left(\eta_{1}-\eta_{2}\right)$ and $z_{2}=\eta_{2} /\left(\eta_{1}-\eta_{2}\right)$ are such that $z_{1}-z_{2}=1$.

Since the local Reynolds number in the interaction region $\rho_{1}^{\prime} U_{1}^{\prime} \ell_{m} / \mu_{1}^{\prime}$ is large, of order $R e^{1 / 2} \gg 1$, the perturbations are governed by the Euler equations, which can be linearized about the base-flow solution to give

$$
\begin{aligned}
& R \frac{\partial u}{\partial x}+U \frac{\partial \rho}{\partial x}+v \frac{\mathrm{d} R}{\mathrm{~d} z}+R \frac{\partial v}{\partial z}=0 \\
& R U \frac{\partial u}{\partial x}+R v \frac{\mathrm{d} U}{\mathrm{~d} z}+\frac{1}{M_{1}^{2}} \frac{\partial p}{\partial x}=0 \\
& R U \frac{\partial v}{\partial x}+\frac{1}{M_{1}^{2}} \frac{\partial p}{\partial z}=0 \\
& U \frac{\partial p}{\partial x}=\frac{U}{R} \frac{\partial \rho}{\partial x}+\frac{v}{R} \frac{\mathrm{d} R}{\mathrm{~d} z}
\end{aligned}
$$

the last expressing the conservation of entropy along any given streamline. The fuel mass fraction and the temperature are also modified by the external pressure perturbation, giving departures that can be described by introducing $Y(z)+$ $\epsilon y(x, z)$ and $T(z)+\epsilon \theta(x, z)$. The perturbation to the mass-fraction field, resulting from the deflection of the stream lines in the interaction region, is determined by integration of

$$
U \frac{\partial y}{\partial x}+v \frac{\mathrm{d} Y}{\mathrm{~d} z}=0
$$

On the other hand, the temperature perturbation $\theta$ can be obtained from the condition of isentropic flow

$$
(\gamma-1) U \frac{\partial p}{\partial x}=\frac{U}{T} \frac{\partial \theta}{\partial x}+\frac{v}{T} \frac{\mathrm{d} T}{\mathrm{~d} z}
$$

or, more directly, from the linearized form of the equation of state

$$
R \theta=\gamma p-T \rho-\left(W_{2}^{-1}-1\right) R T y
$$

in terms of $\rho, p$, and $y$.

As shown by Lighthill [19], the equations (12) can be combined to produce a single equation for the pressure perturbation. The development uses suitable linear combinations of (12a), (12b), and (12d) to yield

$$
\left(1-M^{2}\right) \frac{\partial p}{\partial x}=\frac{\partial}{\partial z}\left(\frac{v}{U}\right)
$$

which leads to

$$
\begin{gathered}
\frac{\partial^{2} p}{\partial z^{2}}+\left(1-M^{2}\right) \frac{\partial^{2} p}{\partial x^{2}}-\frac{\partial \ln M^{2}}{\partial z} \frac{\partial p}{\partial z}=0 \\
\frac{12 \text { of } 35}{\text { American Institute of Aeronautics and Astronautics }}
\end{gathered}
$$


after elimination of $v$ with use made of (12c). From (17) clearly the solution depends fundamentally on the shape of the Mach-number distribution $M(z)$.

Following Lighthill, to simplify the treatment we assume that the mixing layer extends across the finite domain $z_{2}<z<z_{1}$ and that the base flow is uniform outside. The problem then reduces to that of integrating (17) in $z_{2}<z<z_{1}$ subject to the condition that $p$ decays as $x \rightarrow \pm \infty$ and to the additional boundary conditions at $z=z_{1}$ and $z=z_{2}$ obtained from matching with the pressure field in the uniform streams. There, $M^{2}$ is constant, and the pressure-perturbation field obeys the Prandtl-Glauert equation $\partial^{2} p / \partial z^{2}+\left(1-M^{2}\right) \partial^{2} p / \partial x^{2}=0$, which results in a hyperbolic or elliptic differential equation, depending on whether the Mach number is larger or smaller than unity, respectively.

In the supersonic stream, the pressure waves follow real characteristic paths $C_{ \pm}=x \pm \beta_{1} z=$ constant, where $\beta_{1}=\left(M_{1}^{2}-1\right)^{1 / 2}$, with the two solutions having different specific domains of dependence and ranges of influence. The pressure wave in the supersonic zone can be represented by an incident (known) wave, described by $f_{1}\left(x+\beta_{1} z\right)$, and a reflected (unknown) wave, described by $g_{1}\left(x-\beta_{1} z\right)$, so that $p=f_{1}\left(x+\beta_{1} z\right)+g_{1}\left(x-\beta_{1} z\right)$. This outer pressure field is to be employed when defining the boundary condition at $z=z_{1}$, given below for the Fourier analysis in (24). By way of contrast, since (17) is elliptic for subsonic flows, the associated characteristic paths are complex, corresponding to constant values of $x \pm \mathrm{i} \beta_{2} z$, with $\beta_{2}=\left(1-M_{2}^{2}\right)^{1 / 2}$, causing the entire subsonic-flow domain to be the range of influence and domain of dependence. Boundedness of the solution as $z$ approaches $-\infty$ then provides the additional needed boundary condition at $z=z_{2}$, given below in (25) in Fourier space, thereby completing the definition of the pressure-perturbation problem.

The model of the interaction is sketched in Fig. 4, where the incident wave $f_{1}$ that travels along the path $x+$ $\beta_{1} z=$ constant interacts first with the mixing layer at $z=z_{1}$ and $x=-s_{1}=-\int_{0}^{z_{1}}\left(M^{2}-1\right)^{1 / 2} \mathrm{~d} z$. The reflected wave $g_{1}$ leaves the mixing layer following the characteristic $x-\beta_{1} z=$ constant. The functions $g_{1}\left(x-\beta_{1} z\right)$ and $f_{2}\left(x \pm \mathrm{i} \beta_{2} z\right)$ are to be found by analyzing the interaction with the mixing layer for a given function $f_{1}\left(x+\beta_{1} z\right)$.

\section{Solution in Fourier space}

It is useful to transform the partial differential equation (17) into an ordinary differential equation for $z$ by taking a Fourier transform over the $x$ variable. The Fourier-transform pressure $\mathcal{P}(k, z)$ is defined by its inverse transform

$$
p(x, z)=\frac{1}{\sqrt{2 \pi}} \int_{-\infty}^{\infty} \mathrm{e}^{\mathrm{i} k x} \mathcal{P}(k, z) \mathrm{d} k,
$$




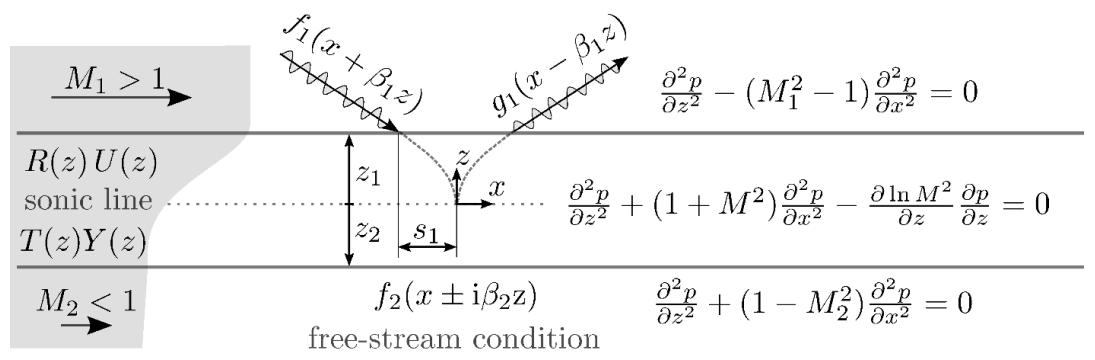

Figure 4. Schematic representation of the incident-wave mixing-layer interaction.

where the independent variable $k$ refers to the Fourier wavenumber of the pressure disturbances. In the formalism selected here, the unitary transformation (18) includes a factor $1 / \sqrt{2 \pi}$ that is not present in Lighthill's boundary-layer analysis, a difference to be kept in mind when comparing the boundary-layer and mixing-layer results. In terms of $\mathcal{P}(k, z)$, the governing perturbation equation inside the mixing layer becomes

$$
\frac{\mathrm{d}^{2} \mathcal{P}}{\mathrm{d} z^{2}}-\frac{\mathrm{d} \ln M^{2}}{\mathrm{~d} z} \frac{\mathrm{d} \mathcal{P}}{\mathrm{d} z}+k^{2}\left(M^{2}-1\right) \mathcal{P}=0
$$

Since $M$ is constant in the supersonic and subsonic streams, in those streams (19) reduces to the equation,

$$
\frac{\mathrm{d}^{2} \mathcal{P}}{\mathrm{d} z^{2}}+k^{2}\left(M^{2}-1\right) \mathcal{P}=0
$$

The solutions to (20) are oscillatory in the supersonic stream $\left(M^{2}>1\right)$ and exponential in the subsonic stream $\left(M^{2}<1\right)$. In the former stream, the pressure field in Fourier space can be written as

$$
\mathcal{P}(k, z)=F_{1}(k) \mathrm{e}^{\mathrm{i} k \beta_{1} z}+G_{1}(k) \mathrm{e}^{-\mathrm{i} k \beta_{1} z},
$$

where $F_{1}(k)$ describes the incident perturbation and $G_{1}(k)$ refers to the corresponding reflected wave. Since the incident perturbation must be prescribed for any given problem, the function $F_{1}(k) \mathrm{e}^{\mathrm{i} k \beta_{1} z}$ is known. The function $G_{1}(k)$, however, is to be determined from (19) by satisfying the boundary condition in the subsonic stream as $z$ approaches negative infinity. Since $G_{1}(k)$ is unknown, the condition (21) must be replaced by a condition that does not involve $G_{1}(k)$. A suitable condition, evident from the derivative of (21), is

$$
\frac{\mathrm{d} \mathcal{P}}{\mathrm{d} z}+\mathrm{i} \beta_{1} k \mathcal{P}=2 \mathrm{i} \beta_{1} k F_{1}(k) \mathrm{e}^{\mathrm{i} k \beta_{1} z}
$$

the right-hand side of which is now a prescribed function. 
For any given value of $k,(19)$ can be integrated numerically from a large positive value of $z$ towards $z=-\infty$, if values of $F_{1}(k)$ and $G_{1}(k)$ are selected. At large negative values of $z$, this solution will approach the solution to the subsonic form of equation (20), which can be written as

$$
\mathcal{P}(k, z)=F_{2}(k) \mathrm{e}^{+|k| \beta_{2} z}+G_{2}(k) \mathrm{e}^{-|k| \beta_{2} z},
$$

with the values of $F_{2}(k)$ and $G_{2}(k)$ determined by the selected values of $F_{1}(k)$ and $G_{1}(k)$ through the integration of (19). Since the solution must, however, be bounded as $z$ approaches negative infinity, $G_{2}(k)$ must vanish. Given $F_{1}(k)$, there will be a value of $G_{1}(k)$ that will result in $G_{2}(k)=0$. This value of $G_{1}(k)$ will correspond to the correct value for the reflected wave. This shooting computational approach will provide $G_{1}(k)$ accurately because any inaccuracies in $G_{1}(k)$ would result in an exponentially divergent solution as $z$ approaches negative infinity.

An alternative to this shooting method is to impose boundary conditions at sufficiently large but finite values of $|z|$, as did Lighthill [19]. If these boundaries are placed sufficiently far, i.e., at values of $|z|$ where equation (20) applies because the Mach number is close to its free-stream values, then the solutions employing (22) and $(23)$ with $G_{2}(k)=0$ at the computational boundaries will be sufficiently accurate. This approach, moreover, facilitates comparisons with the Lighthill solutions. That approach therefore is selected here, with the values of $z_{1}$ and $z_{2}$ associated with the condition of achievement of $99 \%$ of the free-stream Mach number, as previously mentioned.

Applying (22) at $z=z_{1}$ gives

$$
\mathcal{P}_{z}\left(k, z_{1}\right)+\mathrm{i} \beta_{1} k \mathcal{P}\left(k, z_{1}\right)=2 \mathrm{i} \beta_{1} k F_{1}(k) \mathrm{e}^{\mathrm{i} k \beta_{1} z_{1}}
$$

where the subscript $z$ denotes differentiation with respect to this coordinate. In the external subsonic zone, where the pressure distribution (23) holds, the function $G_{2}(k)$ must vanish to avoid a divergent behavior when $z \rightarrow-\infty$, as previously mentioned. Correspondingly, the boundary condition for $\mathcal{P}$ at $z=z_{2}$ becomes

$$
\mathcal{P}_{z}\left(k, z_{2}\right)=\beta_{2}|k| \mathcal{P}\left(k, z_{2}\right)
$$

For a given $F_{1}(k)$, the pressure perturbation in the mixing layer $\mathcal{P}(k, z)$ is obtained by integrating (19) subject to (24) and (25). The Fourier transform of the remaining flow variables can be written in terms of $\mathcal{P}$ and $\mathcal{P}_{z}$. For instance, the functions $\mathcal{Y}(k, z)$ and $\Theta(k, z)$, corresponding to $y(x, z)$ and $\theta(x, z)$ in Fourier space, can be evaluated from

$$
\mathcal{Y}=-\frac{\mathrm{d} Y}{\mathrm{~d} z} \frac{\mathcal{P}_{z}}{k^{2} M^{2}}
$$


and

$$
\Theta=(\gamma-1) T \mathcal{P}-\frac{\mathrm{d} T}{\mathrm{~d} z} \frac{\mathcal{P}_{z}}{k^{2} M^{2}}
$$

obtained from streamwise derivatives of (13) and (14) after (12c) is used to express $\partial v / \partial x$ in terms of the transverse pressure gradient. Also, the solution for $\mathcal{P}(k, z)$ can be used to determine the pressure perturbations in the outer streams, including the reflected wave

$$
G_{1}(k)=\frac{\mathrm{i} k \beta_{1} \mathcal{P}\left(k, z_{1}\right)-\mathcal{P}_{z}\left(k, z_{1}\right)}{2 \mathrm{i} k \beta_{1}} \mathrm{e}^{\mathrm{i} k \beta_{1} z_{1}}
$$

in the supersonic stream, obtained by appropriately eliminating $F_{1}(k)$ after differentiating (21) and evaluating the result at $z=z_{1}$, and the transmitted pressure perturbations

$$
F_{2}(k)=\mathcal{P}\left(k, z_{2}\right) \mathrm{e}^{-|k| \beta_{2} z_{2}}
$$

in the subsonic stream, obtained by evaluating (23) at $z=z_{2}$ with $G_{2}=0$.

\section{Formal solution in Fourier space and limiting asymptotic forms}

The problem of finding $\mathcal{P}(k, z)$ requires numerical integration. The small-scale and large-scale structure of the pressure field can be investigated by considering analytic solutions for large and small values of $|k|$, respectively. To facilitate the analytical development, it is convenient to express the solution formally in terms of two independent orthogonal functions $Q$ and $N$, defined by the solutions to (19) that obey the modified boundary conditions

$$
\begin{aligned}
& Q\left(k, z_{2}\right)=1, \quad Q_{z}\left(k, z_{2}\right)=0, \\
& N\left(k, z_{2}\right)=0, \quad N_{z}\left(k, z_{2}\right)=1 .
\end{aligned}
$$

Using these two independent solutions together with the original boundary conditions (24) and (25) enables the pressure $\mathcal{P}(k, z)$ to be expressed in the form

$$
\frac{\mathcal{P}(k, z)}{F_{1}(k)}=\frac{2 \mathrm{i} \beta_{1} k\left[Q(k, z)+\beta_{2}|k| N(k, z)\right]}{E\left(k, z_{1}, \beta_{1}, \beta_{2}\right)} \mathrm{e}^{\mathrm{i} k \beta_{1} z_{1}}
$$

with $E\left(k, z_{1}, \beta_{1}, \beta_{2}\right)$ given by

$$
E\left(k, z_{1}, \beta_{1}, \beta_{2}\right)=Q_{z}\left(k, z_{1}\right)+\mathrm{i} \beta_{1} k Q\left(k, z_{1}\right)+\beta_{2}|k|\left[N_{z}\left(k, z_{1}\right)+\mathrm{i} \beta_{1} k N\left(k, z_{1}\right)\right] .
$$

In particular, equation (31) exhibits a linear dependence on the external perturbation $F_{1}(k)$ and a more complicated dependence on the Mach-number distribution through the functions $Q(k, z)$ and $N(k, z)$. Solutions will be obtained 
below in the limit $|k| \gg 1$, by using the WKB-like method developed by Langer [33], and also in the limit $|k| \ll 1$, by introducing regular expansions in powers of $k^{2}$ for $Q(k, z)$ and $N(k, z)$. The formal solution (31) is also useful to investigate the upstream influence of the pressure disturbance, whose rate decays for $x \rightarrow-\infty$ as determined by the negative imaginary zero of the denominator of (31) with smallest magnitude $|k|$. This aspect of the solution is to be investigated in Section V, including the differences with the boundary-layer results of Lighthill [19, 20].

\section{A. The limit of large wavenumber}

Consideration of the asymptotic limit $k \gg 1$ allows us to explore the small-scale features of the flow. For the analysis, it is convenient to use, following Ref. [19], the early WKB-like results obtained by Langer [33] for equations of the form (19). According to Langer's analysis, if the two variable coefficients $\mathrm{d}\left(\ln M^{2}\right) / \mathrm{d} \eta$ and $\left(M^{2}-1\right)$ are twice differentiable in the interval $z_{2}<z<z_{1}$, with the latter further satisfying $\left(M^{2}-1\right)>0$ for $z>0$ and $\left(M^{2}-1\right)<0$ for $z<0$, as is the case for the transonic mixing layer, then at leading order in the limit $k \gg 1$ any solution to (19) can be expressed as a linear combination of the functions

$$
\begin{aligned}
& f_{a}=\sqrt{2 \pi} M \frac{|s|^{1 / 6}}{|\beta|^{1 / 2}}(k s)^{1 / 3} J_{-1 / 3}(k s) \\
& f_{b}=\sqrt{2 \pi} M \frac{|s|^{5 / 6}}{|\beta|^{1 / 2}}(k s)^{-1 / 3} J_{1 / 3}(k s) \operatorname{sign}(z)
\end{aligned}
$$

in which

$$
\beta=\left(M^{2}-1\right)^{1 / 2}, \quad \text { and } \quad s=\int_{0}^{z} \beta\left(z^{\prime}\right) \mathrm{d} z^{\prime}
$$

with $z^{\prime}$ being a dummy integration variable and $J_{\nu}$ representing Bessel functions of the first kind. The stretched coordinate $s$ is real in the supersonic domain $z>0$ but imaginary for $z<0$. Its value at $z=z_{1}$ is $s=s_{1}=$ $\int_{0}^{z_{1}} \beta(z) \mathrm{d} z$, while that at the subsonic edge is given by $s=+\mathrm{i} s_{2}$, where

$$
s_{2}=\int_{z_{2}}^{0}\left(1-M^{2}\right)^{1 / 2} \mathrm{~d} z
$$

The formulae (33) simplify for values of $s$ such that $|k s| \gg 1$, corresponding to any fixed transverse location $z$ away from the sonic line as $|k| \rightarrow \infty$, in that the functions $(k s)^{ \pm 1 / 3} J_{\mp 1 / 3}(k s)$ can be replaced by their asymptotic expressions for large values of the argument, leading to

$$
\begin{aligned}
& f_{a}=M \frac{|s|^{1 / 6}}{|\beta|^{1 / 2}}\left[\frac{\mathrm{e}^{\mathrm{i} k s}}{(\mathrm{i} k s)^{1 / 6}}+\frac{\mathrm{e}^{-\mathrm{i} k s}}{(-\mathrm{i} k s)^{1 / 6}}\right] \\
& f_{b}=M \frac{|s|^{5 / 6}}{|\beta|^{1 / 2}}\left[\frac{\mathrm{e}^{\mathrm{i} k s}}{(\mathrm{i} k s)^{5 / 6}}+\frac{\mathrm{e}^{-\mathrm{i} k s}}{(-\mathrm{i} k s)^{5 / 6}}\right] \operatorname{sign}(z) .
\end{aligned}
$$


The constants of integration

$$
\begin{aligned}
& C_{a}^{Q}(k)=\frac{1}{2 \sqrt{3}} \frac{\sqrt{\beta_{2}}}{M_{2}}\left(k^{1 / 6} \mathrm{e}^{k s_{2}}+(-k)^{1 / 6} \mathrm{e}^{-k s_{2}}\right), \\
& C_{b}^{Q}(k)=\frac{1}{2 \sqrt{3}} \frac{\sqrt{\beta_{2}}}{M_{2}}\left(k^{5 / 6} \mathrm{e}^{k s_{2}}+(-k)^{5 / 6} \mathrm{e}^{-k s_{2}}\right), \\
& C_{a}^{N}(k)=\frac{1}{2 \sqrt{3}} \frac{1}{k \sqrt{\beta_{2}} M_{2}}\left(k^{1 / 6} \mathrm{e}^{k s_{2}}-(-k)^{1 / 6} \mathrm{e}^{-k s_{2}}\right), \\
& C_{b}^{N}(k)=\frac{1}{2 \sqrt{3}} \frac{1}{k \sqrt{\beta_{2}} M_{2}}\left(k^{5 / 6} \mathrm{e}^{k s_{2}}-(-k)^{5 / 6} \mathrm{e}^{-k s_{2}}\right),
\end{aligned}
$$

that complete the determination of $Q=C_{a}^{Q} f_{a}+C_{b}^{Q} f_{b}$ and $N=C_{a}^{N} f_{a}+C_{b}^{N} f_{b}$ are obtained by imposing the boundary conditions (30) at the subsonic edge of the mixing layer, with the simplified expressions (36) used in the evaluations, as is appropriate away from the sonic point. These simplified expressions can also be used to evaluate the denominator of (31), which involves values of the functions and their derivatives at the supersonic edge of the mixing layer, while the complete expressions (33) must be used for computing the functions $Q$ and $N$ appearing in the numerator, if a solution valid across the whole mixing layer is to be derived. This evaluation procedure provides

$$
\frac{\mathcal{P}(k, z)}{F_{1}(k)}=\frac{M_{2} \sqrt{\beta_{1}}}{M_{1} \sqrt{\beta_{2}}} \frac{C_{a}^{Q} f_{a}+C_{b}^{Q} f_{b}+\beta_{2}|k|\left(C_{a}^{N} f_{a}+C_{b}^{N} f_{b}\right)}{k \cosh \left(k s_{2}-\mathrm{i} \pi / 4\right)+|k| \sinh \left(k s_{2}-\mathrm{i} \pi / 4\right)} k \mathrm{e}^{\mathrm{i} k\left(\beta_{1} z_{1}-s_{1}\right)}
$$

as a uniformly valid expression for the transverse distribution of pressure perturbation for $k \gg 1$, giving in particular

$$
\frac{\mathcal{P}(k, 0)}{F_{1}(k)}=\frac{2^{1 / 3} \sqrt{\pi}}{3^{2 / 3}\left(-\frac{1}{3}\right) !} \frac{\sqrt{\beta_{1}}}{M_{1}\left[M_{z}(0)\right]^{1 / 6}}[1+\operatorname{isign}(k)]|k|^{1 / 6} \mathrm{e}^{\mathrm{i} k\left(\beta_{1} z_{1}-s_{1}\right)}
$$

for the corresponding variation of the pressure at the sonic line $z=0$, which is seen to exhibit a weak dependence on the local Mach-number gradient $M_{z}(0)=\left.(\mathrm{d} M / \mathrm{d} z)\right|_{z=0}$.

Away from the sonic point (i.e., for $z$ such that $|k s| \gg 1$ ), one may use (36) to evaluate $f_{a}$ and $f_{b}$ in (38), yielding the simplified expressions

$$
\frac{\mathcal{P}(k, z)}{F_{1}(k)}=\sqrt{\frac{\beta_{1}}{\beta}} \frac{M}{M_{1}}\left[\mathrm{e}^{\mathrm{i} k s}+\operatorname{isign}(k) \mathrm{e}^{-\mathrm{i} k s}\right] \mathrm{e}^{\mathrm{i} k\left(\beta_{1} z_{1}-s_{1}\right)},
$$

for $z \gg|k|^{-1}$, where the pressure is oscillatory, and

$$
\frac{\mathcal{P}(k, z)}{F_{1}(k)}=\sqrt{\frac{\beta_{1}}{2|\beta|}} \frac{M}{M_{1}}[1+\operatorname{isign}(k)] \mathrm{e}^{-|k||s|} \mathrm{e}^{\mathrm{i} k\left(\beta_{1} z_{1}-s_{1}\right)},
$$

for $-z \gg|k|^{-1}$, where the pressure decays exponentially for increasing distances from the sonic line. Correspondingly, the large-wave-number components of the reflected and transmitted waves can be obtained by substituting (40) into (28) to give

$$
G_{1}(k)=\operatorname{isign}(k) \mathrm{e}^{2 \mathrm{i} k\left(\beta_{1} z_{1}-s_{1}\right)} F_{1}(k),
$$


and (41) into (29) to give

$$
F_{2}(k)=\sqrt{\frac{\beta_{1}}{2 \beta_{2}}} \frac{M_{2}}{M_{1}}[1+\operatorname{isign}(k)] \mathrm{e}^{|k|\left(\beta_{2} z_{2}-s_{2}\right)} \mathrm{e}^{\mathrm{i} k\left(\beta_{1} z_{1}-s_{1}\right)} F_{1}(k)
$$

In consonance with Riley [23], the results at leading order in the limit $|k| \gg 1$ indicate that the pressure distribution across the mixing layer resulting from the external perturbation becomes largely independent of the boundary condition at the lower edge. Correspondingly, the distribution of $\mathcal{P}$ across the mixing layer, given in (38) for $|k| \gg 1$, is identical to that computed by Lighthill $[19,20]$ for a boundary-layer flow with the same Mach-number distribution $M(z)$, except in a region of characteristic thickness $|k|^{-1}$ near the lower edge, where significant differences appear. As a result, while the pressure perturbation at the sonic line, given in (39), and the shape of the reflected wave, given in (42), are identical for a mixing layer and a boundary layer that have the same $M(z)$ in the supersonic domain, the corresponding pressure at the mixing-layer subsonic boundary, obtained by evaluating (41) at $z=z_{2}$, is half the value predicted by Lighthill at the boundary-layer wall, given by equation (23) in Ref. [20]. Clearly, these differences in pressure magnitude are associated with the different nature of the boundary, with the confinement exerted by the wall resulting in higher pressures.

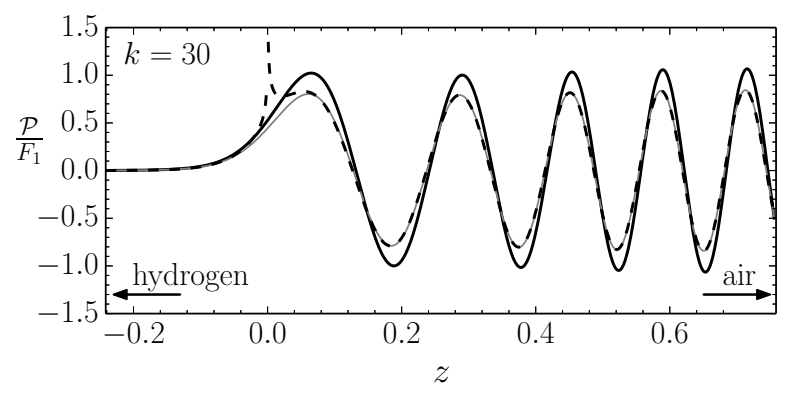

Figure 5. The real part of the function $\mathcal{P} / F_{1}(k)$ as obtained for the hydrogen-air Mach-number distribution of Fig. 3(d) by integration of (19) (thick black solid curves) and by evaluation of the large-wave-number asymptotic predictions given in (38) (thin grey solid curves) and in (40) and (41) (dashed curves) for $k=30$.

The accuracy of the above large-wave-number asymptotic predictions is tested in Fig. 5, which compares the variation with $z$ of the real part of $\mathcal{P} / F_{1}$ obtained by numerical integration of (19) subject to (24) and (25) with that evaluated with use made of (38) for the hydrogen-air mixing layer of Fig. 3(d). For the value $k=30$ selected, the differences are seen to be very small everywhere. The separate predictions given in (40) and (41) for the pressure disturbances in the supersonic and subsonic domains are also included in the plot. As expected, these simplified expressions give a sufficiently accurate description away from the sonic point, but they break down as $M \rightarrow 1$, in the 
region where $|k s|$ is no longer small, where (40) and (41) predict an erroneous pressure divergence resulting from the associated factors $1 / \sqrt{\beta}$ and $1 / \sqrt{|\beta|}$.
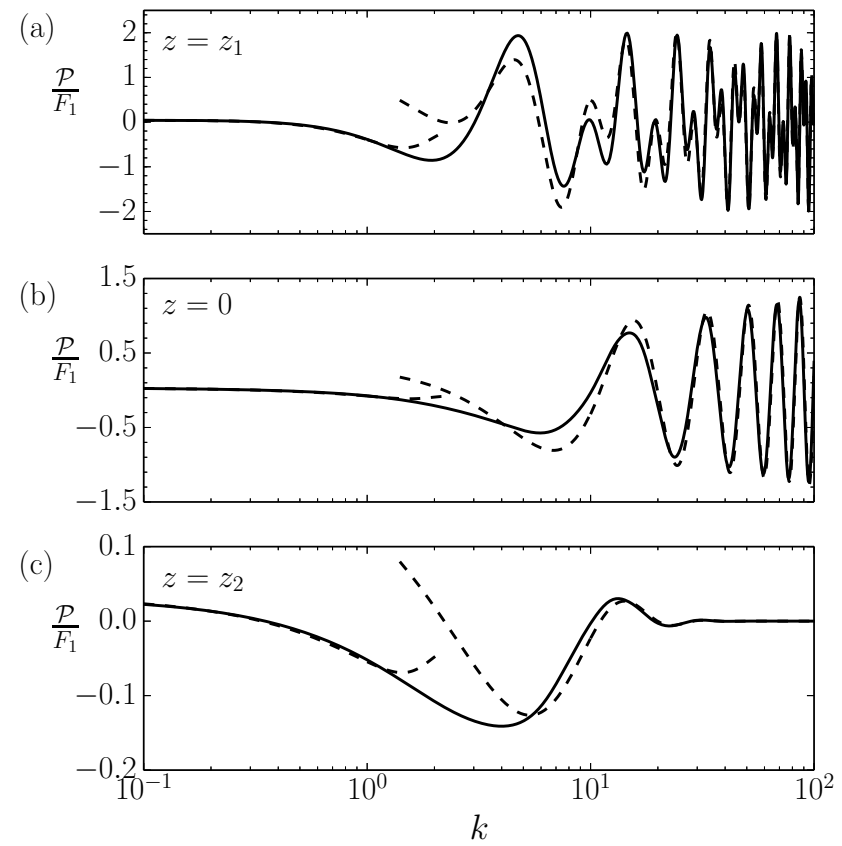

Figure 6. The variation with the wavenumber of the real part of the function $\mathcal{P} / F_{1}(k)$ at the supersonic boundary $z=z_{1}$ (a), at the sonic line $z=0(\mathbf{b})$, and at the subsonic boundary $z=z_{2}$ (c) as obtained for the hydrogen-air Mach-number distribution of Fig. 3(d) by integration of (19) (solid curves) and from the analytical results for large and small wavenumbers (dashed curves).

The asymptotic results are further tested in Fig. 6(a-c), which compares the variation with wavenumber of the real part of $\mathcal{P} / F_{1}$ at $z=\left(z_{1}, 0, z_{2}\right)$ obtained numerically with those determined from evaluations of the analytic predictions, given in (39) for the pressure at the sonic line $z=0$ and in 40 and (41) for the pressure at the supersonic and subsonic mixing-layer boundaries, respectively (results corresponding to the small-wave-number limit, to be discussed below, are also included in the figure). As can be seen, at all three locations the asymptotic predictions remain reasonably accurate until the wavenumber decreases to values of order unity.

The large-wave-number predictions given above can also be used to evaluate the perturbations to the other flow variables, e.g., those of the fuel mass fraction and temperature, given in (26) and (27). As expected, the deflection of the stream lines has a negligible influence on the large-wave-number component of the solution, so that in the limit $|k| \gg 1$ the mass-fraction perturbation $\mathcal{Y}$ vanishes while the temperature perturbation (27) reduces to the local isentropic balance $\Theta / T=(\gamma-1) \mathcal{P}$. 


\section{B. The limit of small wavenumber}

In the opposite limit $|k| \ll 1$, the solution can be obtained by introducing the expansions $Q(k, z)=Q_{0}(z)+k^{2} Q_{1}(z)+$ $\cdots$ and $N(k, z)=N_{0}(z)+k^{2} N_{1}(z)+\cdots$ into (19) and solving sequentially the equations that appear at different orders in powers of $k^{2}$. The development is facilitated by writing (19) in the compact form

$$
\frac{\mathrm{d}}{\mathrm{d} z}\left(M^{-2} \frac{\mathrm{d} \mathcal{P}}{\mathrm{d} z}\right)=k^{2}\left(M^{-2}-1\right) \mathcal{P} .
$$

The right-hand-side term is absent in the equation at leading order

$$
\frac{\mathrm{d}}{\mathrm{d} z}\left(M^{-2} \frac{\mathrm{d} \mathcal{P}_{0}}{\mathrm{~d} z}\right)=0
$$

where $\mathcal{P}_{0}$ is used to denote either one of the leading-order terms $Q_{0}$ and $N_{0}$, so that straightforward integration with boundary conditions $Q_{0}-1=\left(Q_{0}\right)_{z}=0$ and $N_{0}=\left(N_{0}\right)_{z}-1=0$ at $z=z_{2}$ provides

$$
Q_{0}=1 \quad \text { and } \quad N_{0}=\int_{z_{2}}^{z}\left(\frac{M}{M_{2}}\right)^{2} \mathrm{~d} z^{\prime}
$$

At the following order, we find

$$
\frac{\mathrm{d}}{\mathrm{d} z}\left(M^{-2} \frac{\mathrm{d} \mathcal{P}_{1}}{\mathrm{~d} z}\right)=k^{2}\left(M^{-2}-1\right) \mathcal{P}_{0}
$$

thereby providing

$$
Q_{1}=\int_{z_{2}}^{z} M^{2}\left(\int_{z_{2}}^{z^{\prime}}\left(M^{-2}-1\right) \mathrm{d} z^{\prime \prime}\right) \mathrm{d} z^{\prime}
$$

and

$$
N_{1}=\int_{z_{2}}^{z} M^{2}\left(\int_{z_{2}}^{z^{\prime}}\left(M^{-2}-1\right) N_{0} \mathrm{~d} z^{\prime \prime}\right) \mathrm{d} z^{\prime},
$$

upon integration with the homogeneous boundary conditions $Q_{1}=\left(Q_{1}\right)_{z}=N_{1}=\left(N_{1}\right)_{z}=0$ at $z=z_{2}$.

Substitution of the resulting two-term expansions $Q(k, z)=Q_{0}(z)+k^{2} Q_{1}(z)$ and $N(k, z)=N_{0}(z)+k^{2} N_{1}(z)$ into (31) provides an explicit expression for the small-wave-number pressure distribution that is accurate to order $O\left(k^{2}\right)$ across the mixing layer. The resulting prediction at $z=\left(z_{1}, 0, z_{2}\right)$ is compared with the complete numerical results in Fig. 6(a-c). As can be seen, the two-term expansion for $|k| \ll 1$ remains reasonably accurate for values of the wavenumber $|k| \leq 1$.

\section{Upstream decay of the disturbance}

To investigate the upstream propagation of pressure disturbances on the subsonic side of the mixing layer, we adopt the solution strategy utilized by Lighthill [19] for the boundary layer. For $x<0$ the inverse transform $p(x, z)$ of 
(18) can be expressed as $-\sqrt{2 \pi} i$ times the sum of the residues of $\mathcal{P}(k, z) \mathrm{e}^{\mathrm{i} k x}$ at the zeros of the denominator of (31) in the lower half of the complex $k$ plane, which are located along the imaginary axis (i.e., $k=-\mathrm{i} \kappa_{0},-\mathrm{i} \kappa_{1},-\mathrm{i} \kappa_{2}, \ldots$ with $\left.\kappa_{0}<\kappa_{1}<\kappa_{2} \cdots\right)$. The dominant term in the far field, corresponding to large values of $-x$, is that associated with the smallest zero, $k=-\mathrm{i} \kappa_{0}$. Correspondingly, the product of the inverse logarithmic decrement, $\kappa_{0}^{-1}$, and the mixing-layer thickness $\ell_{m}$ provides a measure for the effective distance of upstream influence.

To determine $\kappa_{0}$, it is convenient to introduce $k=-\mathrm{i} \kappa$ in the denominator of (31) to yield

$$
Q_{z}\left(-\mathrm{i} \kappa, z_{1}\right)+\beta_{1} \kappa Q\left(-\mathrm{i} \kappa, z_{1}\right)+\beta_{2} \kappa\left[N_{z}\left(-\mathrm{i} \kappa, z_{1}\right)-\beta_{1} \kappa N\left(-\mathrm{i} \kappa, z_{1}\right)\right]=0
$$

where the functions $Q$ and $N$ are obtained by integration of (19) with boundary conditions (30). For a given Mach number distribution, the numerical solution to (50) provides a discrete number of real positive zeros $\kappa_{n}$ of increasing magnitude. For instance, when the profiles $M(z)$ shown in Fig. 3(c,d) are used in the computation of $Q(k, z)$ and $N(k, z)$, one obtains for the first three zeros from (50) the values $\kappa_{0}=4.7, \kappa_{1}=16.34$, and $\kappa_{2}=27.23$ for the ethylene-air mixing layer and the values $\kappa_{0}=7.32, \kappa_{1}=27.6$, and $\kappa_{2}=45.22$ for the hydrogen-air mixing layer.

The results indicate that, for mixing layers, the decay of the perturbation is quite rapid, because $\kappa_{0}$ is moderately large, so that the pressure disturbance is only felt at distances of the order of a fraction of the mixing-layer thickness. This is in contrast with the results previously obtained for the boundary layer, in which the decay was seen to be very slow [20], with perturbations reaching far upstream but it agrees with the results of Riley [23]. Since $\kappa_{0}$ was very small for the boundary layer, the limit of small wavenumbers was correspondingly used by Lighthill [20] to determine approximate analytic expressions for $\kappa_{0}^{-1}$. In the present problem, however, the numerical results suggest that the opposite limit $|k| \gg 1$ should be considered instead, with the value of $\kappa_{0}$ obtained from the analysis of the zeros of the denominator in (38), similar to what was done by Riley [23]. Introducing $k=-\mathrm{i} \kappa$ leads to $\tan \left(\kappa s_{2}+\pi / 4\right)=-1$, which can be solved to give

$$
\kappa_{n}=\frac{\pi}{2}(1+2 n) s_{2}^{-1}
$$

where $s_{2}$, defined in (35), carries a dependence on the Mach-number distribution across the subsonic layer. Using in (51) the values $s_{2}=0.29$ and $s_{2}=0.175$ corresponding to the ethylene-air and to the hydrogen-air mixing layers, respectively, provides the values $\kappa_{0}=5.45, \kappa_{1}=16.34$, and $\kappa_{2}=27.23$ and $\kappa_{0}=8.98, \kappa_{1}=26.95$, and $\kappa_{2}=44.92$ for the first three zeros. As can be seen by comparing these values with the numerical results, the accuracy of (51) improves for larger $\kappa$, an expected result. The approximation $\kappa_{0}=\pi /\left(2 s_{2}\right)$ that follows from (51) overpredicts the first zero by about $20 \%$ for the two mixing layers considered here. As the Mach number of the subsonic stream 
decreases, the accuracies of these approximations decrease, approaching results like those of Lighthill [20].

It is worth mentioning that, while the inverse logarithmic decrement $\kappa_{0}^{-1}$ in boundary layers is found to be approximately proportional to the square of the supersonic Mach number $M_{1}^{2}$ [20], in mixing layers the large-wave-number analysis provides a value $\kappa_{0}^{-1}=2 s_{2} / \pi$ entirely independent of the Mach-number distribution in the supersonic stream. Since $s_{2}$ is proportional to $\left|z_{2}\right|$, the characteristic distance of upstream influence $\kappa_{0}^{-1} \ell_{m}$ becomes proportional to the thickness of the subsonic portion of the mixing layer, which is seen in Fig. 3(c,d) to be markedly smaller for the hydrogen-air mixing layer, as was indicated previously. The differences in profiles of $M(z)$ shown in Fig. 3(c,d) also indicate that consideration of a sufficiently accurate molecular transport model is essential in computing $s_{2}$ accurately, so that, for instance, Soret effects cannot be neglected when dealing with hydrogen.

\section{Interaction with a weak shock}

The above theory applies to the interaction of any given weak external pressure perturbation with a transonic mixing layer. The specific response to a weak shock can be investigated by considering an incident pressure jump defined by the Heaviside step function $f_{1}=\mathcal{H}\left(x+\beta_{1} z+s_{1}-\beta_{1} z_{1}\right)$, whose Fourier transform is given by $F_{1}(k)=$ $(\pi \delta(k)+1 / \mathrm{i} k) \exp \left[\mathrm{i} k\left(s_{1}-\beta_{1} z_{1}\right)\right] / \sqrt{2 \pi}$. The resulting distribution of pressure perturbation $p(x, z)$ across the mixing layer can be obtained from the inverse transformation (18) once the Fourier pressure function $\mathcal{P}(k, z)$ is determined by integration of (19) subject to (24) and (25). Since the response to the pressure discontinuity is anticipated to have a dominant large-wave-number component at distances $x$ of order unity, the simplified results obtained above in the limit $|k| \gg 1$ can be used in the analysis of the solution in the interaction region. The computation of the pressure is still far from trivial, since it involves the cumbersome task of inverting the Bessel-type functions present in (38) when use is made of (33). The solution is facilitated by working with the simplified expressions (40) and (41), which hold away from the sonic point in the supersonic and subsonic domains, respectively, yielding

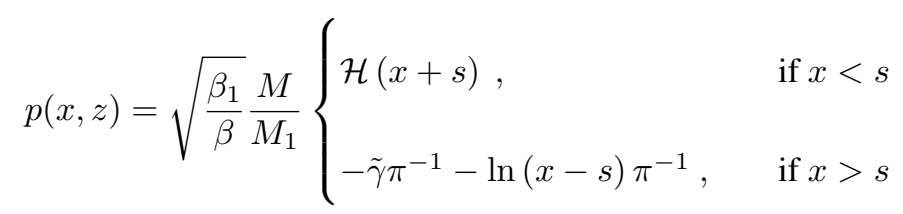

and

$$
p(x, z)=\frac{1}{\pi} \sqrt{\frac{\beta_{1}}{2|\beta|}} \frac{M}{M_{1}}\left[\frac{\pi}{2}-\tilde{\gamma}-\ln \left(\sqrt{|s|^{2}+x^{2}}\right)-\tan ^{-1}\left(\frac{x}{|s|}\right)\right]
$$

upon application of the inverse Fourier transform. The symbol $\tilde{\gamma}$ refers to the Euler-Mascheroni constant. These pressure functions are not valid in the vicinity of the sonic line $z=0$, where they should be replaced by the inverse 
transform of the Fourier pressure (38). In particular, its value at $z=0$, given in (39), can be used to derive

$$
p(x, 0)=\frac{\sqrt{\beta_{1}}}{\sqrt{2} M_{1}\left[M_{z}(0)\right]^{1 / 6}}\left[\frac{6^{1 / 3}(1 / 6) ! \sqrt{2+\sqrt{3}}}{(-1 / 3) !}\right]\left[1+\frac{1-(2-\sqrt{3}) \operatorname{sign}(x)}{|x|^{1 / 6}}\right],
$$

for the pressure perturbation along the sonic line.

The supersonic-side pressure distribution (52) comprises two different waves, namely, the incident perturbation and a reflected wave. The former is just the incident step pressure wave that follows the path $x=-s(z)$, with an amplitude proportional to $M /\left(M^{2}-1\right)^{1 / 4}$. On reflecting from the sonic line, the step changes its character to give a positive logarithmic infinity, i.e., a sudden compression followed by an expansion zone, that propagates outwards along the characteristic $x=s(z)$. As the point of incidence $(x, z)=(0,0)$ is approached, (52) ceases to be valid. The step and logarithmic singularities are seen to merge in the near-sonic region, leading to an algebraic singularity, with the pressure diverging proportional to $\left|M_{z}(0) x\right|^{-1 / 6}$, as can be seen in (54). In the subsonic layer, the solution given in (53) is regular for any nonzero value of $z<0$. The solution is completed by the reflected wave in the outer supersonic zone

$$
g_{1}(x, z)=-\tilde{\gamma}-\frac{1}{\pi} \ln \left[x-\beta_{1} z+\beta_{1} z_{1}-s_{1}\right]
$$

which can be obtained directly by the Fourier inversion of (42) (or simply by setting $z=z_{1}$ in the right-traveling wave in (52)), and by the wave transmitted into the subsonic side,

$$
f_{2}(x, z)=\frac{1}{\pi} \sqrt{\frac{\beta_{1}}{2 \beta_{2}}} \frac{M_{2}}{M_{1}}\left\{\frac{\pi}{2}-\tilde{\gamma}-\tan ^{-1}\left(\frac{x}{s_{2}+\beta_{2} z_{2}-\beta_{2} z}\right)-\ln \left[\sqrt{\left(s_{2}+\beta_{2} z_{2}-\beta_{2} z\right)^{2}+x^{2}}\right]\right\}
$$

obtained with use made of (53). Note that the logarithmic nature of $g_{1}$ corresponds to that of the mixing-layer pressure wave along the right-running characteristic $x=-s(z)$.

The pressure perturbations given in (52) and (53) for the supersonic and subsonic domains and the intermediate sonic-line pressure distribution 54 are represented in Fig. 7(a-f) for the ethylene-air and hydrogen-air mixing layers. The trajectories of the incident and reflected waves in the supersonic stream and the distributed pressure disturbances in the subsonic stream are qualitatively similar for both mixing layers, although quantitative differences arise from the associated differences in Mach-number distribution displayed in Fig. 2(c,d) . Along the sonic line, however, the streamwise pressure distributions are practically indistinguishable, because the values of $\left[M_{z}(0)\right]^{1 / 6}$ in $(54)$ happen to be approximately equal for these two configurations. Since in the limit of $|k| \gg 1$ the functions $\mathcal{P}$ and $\Theta$ are proportional, as dictated by (27), the corresponding temperature-perturbation field $\theta(x, z)$ would satisfy $\theta=(\gamma-1) T p$, thereby giving a spatial distribution qualitatively similar to that shown in Fig. 6 for the pressure-perturbation field. 


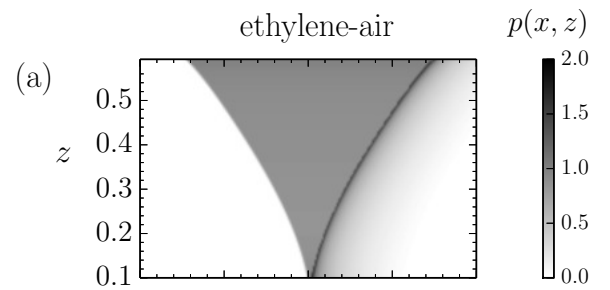

$$
\begin{aligned}
& z) \\
& 2.0 \\
& 1.5 \\
& 1.0 \\
& 0.5 \\
& 0.0
\end{aligned}
$$

(c)
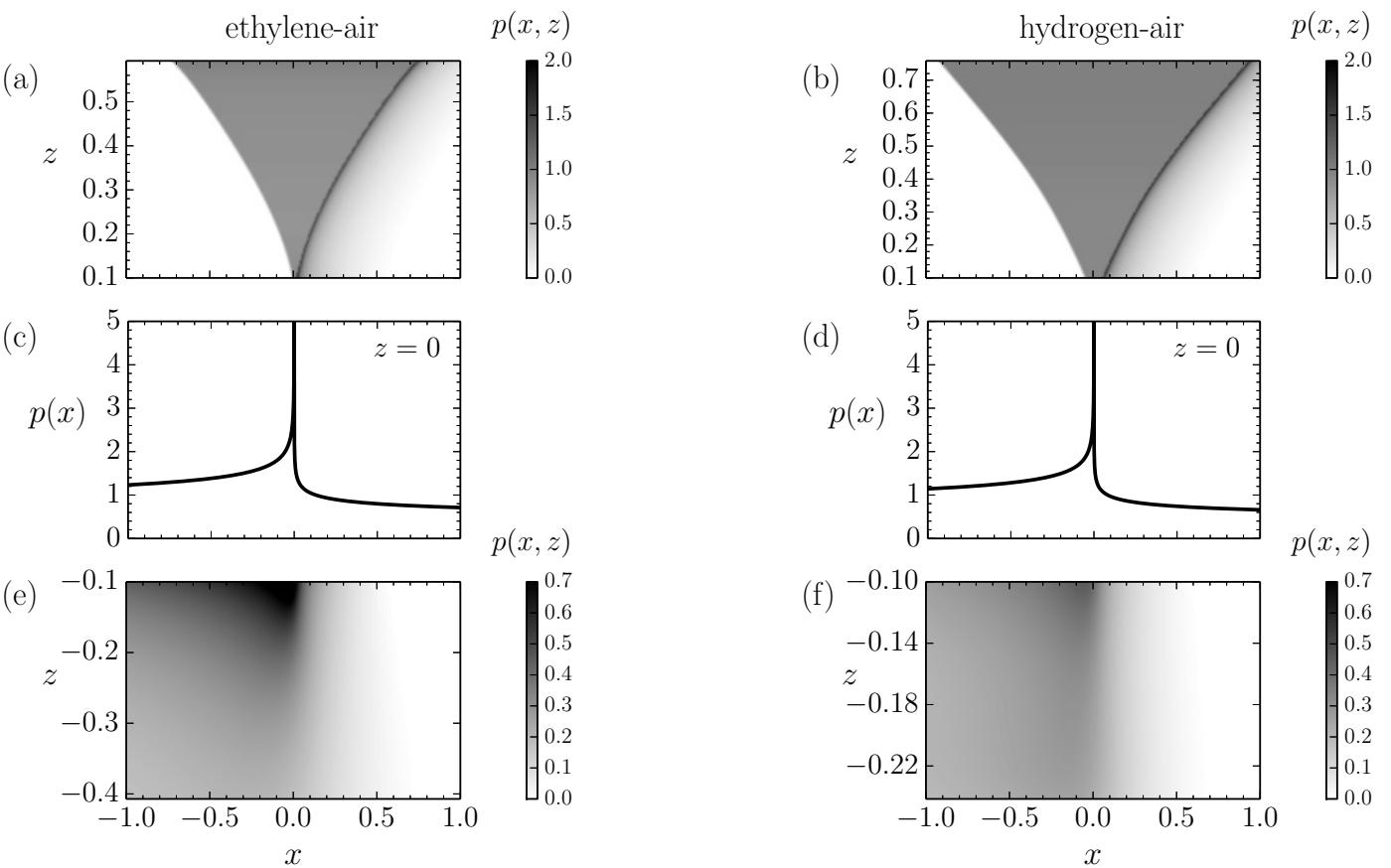

(d)
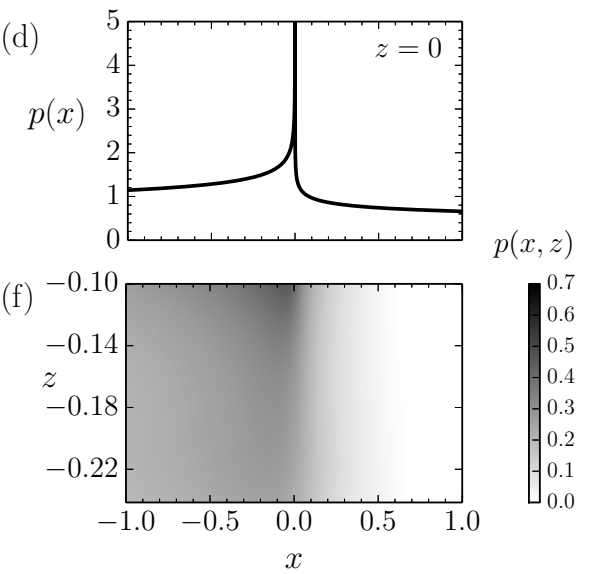

Figure 7. The large-wave-number pressure perturbation caused by a weak shock as obtained from evaluation of (a,b) equation (52) for the supersonic side, (c,d) equation (54) corresponding to the sonic line, and (e,f) equation (53) for the subsonic side, for the ethylene-air (left-hand-side plots) and hydrogen-air (right-hand-side plots) Mach-number distributions of Fig. 3(c,d). 
The logarithmic singularity of the reflected wave and the algebraic singularity $|x|^{-1 / 6}$ at the point of incidence, both of which also are present in the original boundary-layer analysis of Lighthill [19], appear to be inconsistent with the hypothesis of small disturbances. They emerge in the linear theory as a consequence of the discontinuous nature of the incident pressure wave. It is naturally expected that, in realistic configurations, the singularity would disappear as a consequence of nonlinear effects acting locally, leading to a pressure field that would be similar to that depicted in Fig. 7(a,f), except near the singularities, where the infinities would be replaced by large but finite values of the pressure. As noted by Lighthill [19] for boundary-layer flows, this view seems to be supported by experimental observations [34, 35, 36, 37].

In the framework of the linear theory, the pressure singularity can be removed by accounting for the finite thickness of the incident weak shock, an approach that is motivated by the fact that the shock thickness is inversely proportional to the shock strength [38]. As a simplified example, one may consider external perturbations with a piecewise linear pressure distribution

$$
f_{1}(x, z)=\frac{\left(x_{+}\right) \mathcal{H}\left(x_{+}\right)-\left(x_{+}-\ell_{s}\right) \mathcal{H}\left(x_{+}-\ell_{s}\right)}{\ell_{s}}
$$

where $x_{+}=x+\beta_{1} z+s_{1}-\beta_{1} z_{1}$ is the incident-wave path and $\ell_{s}$ is the ratio of the shock-wave thickness to the mixing-layer thickness $\ell_{m}$. The corresponding Fourier transform

$$
F_{1}(k)=\left[\frac{1-\mathrm{e}^{\mathrm{i} k \ell_{s}}}{k^{2}}+\ell_{s} \pi \delta(k)\right] \frac{\mathrm{e}^{\mathrm{i} k\left(s_{1}-\beta_{1} z_{1}\right)}}{\ell_{s} \sqrt{2 \pi}},
$$

can be used in the large-wave-number prediction (40) to generate from (18) the pressure distribution

$$
p(x, z)=\sqrt{\frac{\beta_{1}}{\beta}} \frac{M}{M_{1}}\left[\frac{(x+s) \mathcal{H}(x+s)-\left(x+s-\ell_{s}\right) \mathcal{H}\left(x+s-\ell_{s}\right)}{\ell_{s}}\right]
$$

if $x<s$, and

$$
p(x, z)=\sqrt{\frac{\beta_{1}}{\beta}} \frac{M}{M_{1}}\left[-\frac{\tilde{\gamma}+1}{\pi}-\frac{(x-s) \ln (x-s)-\left(x-s-\ell_{s}\right) \ln \left(x-s-\ell_{s}\right)}{\pi \ell_{s}}\right]
$$

if $x>s$, which, unlike (52), yields a finite value of $p$ along the right-running characteristic $x=-s(z)$. Similarly, contrary to their counterparts (54) and (55), the corresponding expressions for the pressure distribution along the sonic line

$$
\begin{aligned}
& p(x, 0)=\frac{\sqrt{\beta_{1}}}{\sqrt{2} \ell_{s} M_{1}\left[M_{z}(0)\right]^{1 / 6}} \frac{6^{1 / 3}(-5 / 6) ! \sqrt{2+\sqrt{3}}}{5(-1 / 3) !} \\
& \times\left[(2-\sqrt{3})\left(\left|x-\ell_{s}\right|^{5 / 6}-|x|^{5 / 6}\right)-\left|x-\ell_{s}\right|^{5 / 6} \operatorname{sign}\left(x-\ell_{s}\right)+|x|^{5 / 6} \operatorname{sign}(x)\right]
\end{aligned}
$$


and the associated reflected shock, that follows the path $x_{-}=x+\beta_{1} z_{1}-s_{1}-\beta_{1} z$,

$$
g_{1}(x, z)=-\frac{\tilde{\gamma}+1}{\pi}-\frac{\left(x_{-}\right) \ln \left(x_{-}\right)-\left(x_{-}-\ell_{s}\right) \ln \left(x_{-}-\ell_{s}\right)}{\pi \ell_{s}}
$$

are free from singularities, with the infinities in pressure present in (54) and (55) being replaced by large peak values of order $\ell_{s}^{-1 / 6}$ in (61) and of order $\ln \left(\ell_{s}^{-1}\right)$ in (62). These results indicate that the singularities of the infinitesimally thin shock translate into a ridge when the finite thickness of the shock is considered.

\section{Vorticity production}

Although, unlike the work of Buttsworth [25], only weak shocks are addressed in this study, the results can be used to examine trends of induced effects in applications involving shock-wave ignition of fuel-air mixing-layer flows, of interest for combustion processes in supersonic engines. For example, the direct effects of the incident pressure wave on the fuel and temperature distributions are described by (26) and (27), giving results that could be incorporated as perturbations in computations of ignition distances by integration of the flow equations downstream from the interaction region. The results would be qualitatively indicative of the influences of stronger shocks.

The interaction of the pressure wave leads to an additional indirect (although possibly important) effect associated with the local generation of vorticity, which may promote the instability of the mixing-layer flow, thereby enhancing the combustion rate by increasing the downstream mixing rate of the two streams. Alternatively, this effect could also reduce the vorticity and thereby inhibit instability and its associated turbulent mixing. In examining vorticity production, it is convenient to express the perturbed nondimensional vorticity, scaled with its characteristic value $U_{1}^{\prime} / \ell_{m}$, in the form $U_{z}(z)+\varepsilon \omega(x, z)$. Here $U_{z}$, clearly positive in the mixing layer, corresponds to a positive baseflow vorticity according to the conventional right-hand rule, this being the component of the vorticity vector in the direction pointing into the paper in Fig. 2. Positive values of $\omega$ therefore increase the vorticity and thus presumably enhance the instability of the laminar mixing layers, leading to earlier onset of turbulence along with increased overall mixing rates.

The equation for $\omega=\partial u / \partial z-\partial v / \partial x$ can be shown from (12) to be given by

$$
U \frac{\partial \omega}{\partial x}+v U_{z z}=U_{z}\left(\frac{\partial u}{\partial x}+\frac{\partial v}{\partial z}\right)-\frac{1}{M_{1}^{2}} \frac{R_{z}}{R^{2}} \frac{\partial p}{\partial x}
$$

which indicates that, along the perturbed stream line, the vorticity changes through the combined effects of variabledensity flow stretching and baroclinic torque, the two source terms on the right-hand side of (63). The former arises as 
a result of the interaction of the induced dilatation rate with the background shear, while the latter is the result of the nonalignment of the induced pressure gradient and the background density gradient. The second term on the left-hand side of (63) emerges because of the deflection of the stream lines, its magnitude being proportional to the curvature of the base-flow velocity $U_{z z}$.

The vorticity equation (63) can be expressed in a more compact form by using (1) together with

$$
\frac{\partial u}{\partial x}+\frac{\partial v}{\partial z}=-U \frac{\partial p}{\partial x}
$$

obtained from a straightforward combination of (12a) and (12d), to yield

$$
\frac{\partial \omega}{\partial x}=-\Omega \frac{\partial p}{\partial x}-\frac{U_{z z}}{U} v
$$

where the vorticity-production factor

$$
\Omega(z)=U_{z}+\frac{U}{M^{2}} \frac{R_{z}}{R}
$$

measures the collective effects of flow stretching and baroclinic torque. Taking the streamwise derivative of (65) and expressing the result in Fourier space, after using (12c) to eliminate $\partial v / \partial x$, yields

$$
\varpi=-\Omega \mathcal{P}-U_{z z} \frac{\mathcal{P}_{z}}{k^{2} M^{2}}
$$

for the transform $\varpi(k, z)$ of the vorticity perturbation, defined by its inverse transform

$$
\omega(x, z)=(2 \pi)^{-1 / 2} \int_{-\infty}^{\infty} \mathrm{e}^{\mathrm{i} k x} \varpi(k, z) \mathrm{d} k .
$$

Equation (67) can be used to evaluate $\varpi$ from the results of the pressure-perturbation transform $\mathcal{P}$ and its derivative $\mathcal{P}_{z}$, together with the transverse distributions of $\Omega(z), U_{z z}(z)$, and $M(z)$. For large wavenumbers $|k| \gg 1$, the streamline deflection is seen from (67) to produce a negligible influence, and the resulting vorticity field becomes linearly proportional to the pressure perturbation, with $\Omega$ entering as a proportionality factor.

Profiles of $\Omega(z)$ and its stretching and baroclinic contributions corresponding to the mixing layers of Fig. 2(a-d) are shown in Fig. 8(a,b). For the hydrogen-air mixing layer, both $U_{z}$ and $R_{z}$ are positive, with the result that flow stretching and baroclinic torque cooperate to create vorticity of the same sign. The resulting function $\Omega$ is everywhere positive and shows a prominent peak in the subsonic stream near the sonic line. For mixing layers with constant molecular weight approximating ethylene-air, however, $R_{z}<0$, because the density is inversely proportional to the temperature and the air stream is hotter. In this case, the competition of flow stretching and baroclinic torque causes the resulting 

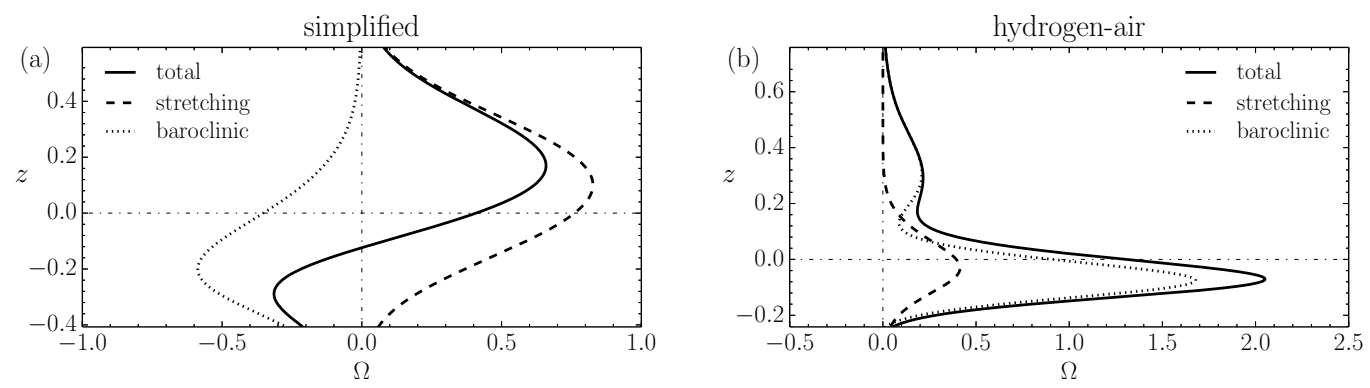

Figure 8. The vorticity-production factor $\Omega$ (solid lines) along with the stretching (dashed lines) and baroclinic (dotted lines) contributions as a function of the dimensionless coordinate $z$ for the ethylene-air and hydrogen-air mixing layers of Fig. 2(a-d).

function $\Omega$, shown on Fig. 8(b), to be predominantly negative in the subsonic domain, where the baroclinic torque is dominant, and positive in the supersonic domain, where flow stretching prevails. Its magnitude is significantly smaller than that corresponding to hydrogen-air mixing layers, suggesting that the perturbations to the vorticity field are more important for the latter, especially in the vicinity of the sonic line. Clearly, many of these quantitative results depend on the specific set of boundary conditions selected for the mixing-layer problem. For example, consideration of smaller fuel-side temperatures $T_{2}$ is seen to extend the range of negative $\Omega$ in the ethylene-air mixing layer. Likewise, for sufficiently small $T_{2}$, a region of negative $R_{z}$ appears in the hydrogen-air mixing layer near the subsonic boundary, where $\Omega$ may become negative.

According to (65), vorticity can be either created or destroyed depending on the sign of the product $-\Omega \partial p / \partial x$, the $U_{z z}$ term being smaller. This possibility of creation or destruction also occurs for finite-amplitude waves in purely supersonic flow [25]. It can be concluded from the pressure fields shown in Fig. 6 that at any given transverse location $z$ there is an upstream region of adverse pressure gradient $(\partial p / \partial x>0)$, including a finite pressure jump across the shock in the supersonic stream, and a downstream region of favorable pressure gradient $(\partial p / \partial x<0)$. For the hydrogen-air mixing-layer result in Fig. 7(b), for which $\Omega$ is positive everywhere, vorticity is destroyed upstream and produced downstream all across the mixing layer. This interaction thus may tend to delay the transition to turbulence ahead of shock impingement in this case. For the other case considered, however, the sequence is reversed in the subsonic domain with $\Omega<0$, where there is vorticity production in the upstream region of adverse pressure gradient followed by vorticity destruction in the downstream region with $\partial p / \partial x<0$. The effect of the shock on mixing-layer transition thus will be different for different fuels and in different flows. These qualitative observations may be of help in attempts to tailor flows to affect mixing. 


\section{Conclusions}

A notable finding of this investigation is the relative simplicity of shock interaction with transonic mixing layers, in comparison with its interaction with boundary layers on walls, as studied by Lighthill [19, 20]. The triple-deck analysis that underlies the latter problem reduces to just two decks for this mixing layer. The reason for this simplification is that the Mach number of the subsonic stream does not become small. The present analysis applies for a wide range of Mach numbers of order unity, but it fails if the subsonic-stream Mach number becomes small compared with unity.

Although the same generalized Prandtl-Glauert equation applies in the transonic region, a number of differences in conclusions arise from the fact that the appropriate asymptotic analyses for large Reynolds numbers in laminar flows differ in this transonic case. For example, while Lighthill found that the pressure disturbances in the boundary layer make themselves felt far upstream from the point of shock-wave impingement on the wall, in the transonic mixing layer those influences are restricted to a small region, having a streamwise distance of the same order as the thickness of the mixing layer. As a consequence, in the context of the Fourier transform in the streamwise direction, the WKB asymptotic expansions for large values of the streamwise wavenumber provide more accurate results for variations of pressure-wave influences in the flow direction than do corresponding regular expansions for small values, in transonic problems.

In this weak-shock limit, which decouples the underlying mixing-layer base flow from the pressure-interaction analysis, pressure-wave interactions modify the mixing layer appreciably only in a region extending upstream and downstream for a distance of the order of the mixing-layer thickness or less. It was found that an incident step-function pressure wave curves and tends to become normal to the flow direction at the sonic line, its discontinuity disappearing there but generating a distributed pressure field in the subsonic region. In addition, the sharp-fronted incident pressure wave generates a reflected wave that follows an approximately mirror-image trajectory in the supersonic stream but possesses a distributed rather than sharp structure, exhibiting a logarithmic profile that builds rapidly to pressures in excess of that of the incident wave but then slowly decays, reminiscent of a rarefaction. Even though the derived structures and wave dynamics pertain specifically to linear theory, similar behaviors would be expected for sufficiently weak finite-amplitude shocks, so long as their strength is not great enough to alter the incoming subsonic portion of the mixing layer significantly. Methods of Riley [23] and Buttsworth [25] can address finite-amplitude influences in transonic and supersonic mixing layers, respectively.

Although these general attributes are common to all interactions of sufficiently weak pressure waves with transonic 
mixing layers, there are notable differences that depend on differences in the properties of ideal gas mixtures that are assumed to be present in each of the two streams. The structures of the transonic mixing layers themselves differ, depending on the composition of each stream, even before any pressure-wave interaction. The two specific cases investigated here correspond to both streams effectively having the properties of air and to the supersonic stream being air and the subsonic stream hydrogen. In the former case, more relevant for fuels such as ethylene, velocity, temperature, and composition profiles are approximately symmetrical across the mixing layer, while with hydrogen they are not, indicating different behaviors of these two SCRAMJET fuels.

The detailed consideration of the hydrogen case, motivated by interest in supersonic combustion, pertains to conditions in which the air stream is appreciably hotter than the hydrogen stream, as occurs in such applications. In that case, property variation across the mixing layer, particularly the profile of the Mach number, which is of primary relevance to the pressure-wave interactions, are especially asymmetrical, monotonic but exhibiting three points of inflection. Moreover, Soret diffusion exerts a strong influence on such profiles, while Dufour effects remain negligible. The presence of hydrogen also leads to an appreciably thicker mixing layer because of its large molecular diffusivity, with the sonic point occurring closer to the hydrogen side, for a fixed Mach-number ratio of the two streams, because of its higher sound speed. These variations clearly depend strongly on the specific conditions selected for the analysis, and different profiles, in some cases even possibly non-monotonic, would be encountered under other conditions.

The different structures of the mixing layers lead an incoming pressure wave to induce different modifications to those structures. For example, the modifications to the profiles of the temperature and composition fields are different in the two different cases analyzed. This will be significant in future considerations of influences of the incident waves on autoignition times in the mixing layers. Here, besides deriving the resulting temperature, pressure, and fuel-mass fraction fields, we examined, in particular, the different influences on modifications of vorticity profiles, with the thought that increased vorticity enhances instabilities that result in transition to turbulence, beneficial to subsequent combustion, by introducing a turbulent contribution to the mixing.

Two physical phenomena were found to modify the vorticity fields, namely the baroclinic torque produced by the pressure-gradient variation and the action of the local base-flow strain rate on the dilatation that is produced by the incident disturbance. A non-dimensional function of the base-flow profiles was identified that quantifies the magnitude of the vorticity-field modifications, including both of these effects.

It was found that the incident perturbations could increase the vorticity in portions of the mixing layers and decrease 
it in other portions. For example, in the mixing layer with air properties on both sides, the relevant parameter is positive in the supersonic part of the mixing layer and negative in the subsonic part. The parameter was found to be positive everywhere across the mixing layer for the particular hydrogen case analyzed, although that need not be true always for hydrogen. It does, however, appear that while the baroclinic and dilatation effects tend to be of opposite sign for mixing layers in which both streams have similar properties, they both tend to be positive with hydrogen in one stream, yet another difference for different SCRAMJET fuels. Upstream from the impingement point, where the modifications to the vorticity generally are the largest, the effect is to reduce vorticity levels in the hydrogen-air mixing layer.

Ongoing extensions of this work include the consideration of non-linear interactions of mixing layers with finiteamplitude shock waves that occur in SCRAMJETS $[6,7,9,10,11,12]$. In addition, particularly for the hydrogen-air case considered here, it is worthwhile to proceed to analyze the autoignition occurring in the laminar mixing layer, to determine the extent to which pressure perturbations incident from the supersonic stream may augment ignition, thereby reducing ignition distances. Further extensions of this work also include consideration of shock-generated disturbances on steady combustion of diffusion flames. For all of these problems, turbulence is a complicating effect for which it could be misleading to try to extend the present results by simplified methods such as merely replacing laminar diffusivities by turbulent diffusivities, since shock-turbulence interactions can be complex. Related problems for turbulent mixing layers therefore deserve extensive further study.

\section{Acknowledgments}

This work was supported by the US AFOSR Grants \# FA9550-12-1-0138 and \# FA9550-14-1-0219. We are grateful to Amable Liñán for useful conversations at the early stages of this project.

\section{References}

[1] Ferri, A., "Mixing-controlled supersonic combustion,” Ann. Rev. Fluid Mech., Vol. 5, 1973, pp. 301-338.

[2] Andreopoulos, Y., Agui, J. H., and Briassulis, G., "Shock wave-turbulence interactions," Ann. Rev. Fluid Mech., Vol. 32, 2000, pp. 309-345.

[3] Dolling, D. S., "Fifty years of shock-wave/boundary-layer interaction research: what next?," AIAA Journal, Vol. 39, No. 8, 2001, pp. 1517-1531. 
[4] Jammalamadaka, A., Li, Z., and Jaberi, F., "Numerical investigations of shock wave interactions with a supersonic turbulent boundary layer," Phys. Fluids, Vol. 26, 2014, 056101.

[5] Pirozzoli, S. and Bernardini, M., "Direct Numerical Simulation Database for Impinging Shock Wave/Turbulent Boundary-Layer Interaction,” AIAA Journal, Vol. 29, No. 6, 2011, pp. 1307-1312.

[6] Waidmann, W., Alff, F., Brummund, U., Böhm, M., Clauss, W., and Oschwald M., "Experimental investigation of the combustion process in a supersonic combustion ramjet (SCRAMJET),” In: DLR Jahrestagung, pp. 629-638, Erlangen, 1994

[7] Laurence, S. J., Karl, S., Schramm, J., Martínez, J. and Hannemann, K., “Transient fluid-combustion phenomena in a model scramjet," J. Fluid Mech., Vol. 722, 2013, pp. 85-120.

[8] Gutmark, E. J., Schadow, K. C., and Yu, K. H., "Mixing enhancement in supersonic free shear flows," Ann. Rev. Fluid Mech., Vol. 27, 1995, pp. 375-417.

[9] Menon, S., “Shock-wave-induced mixing enhancement in scramjet combustors," AIAA Paper 89-0104, 1989.

[10] Lu, P. J. and Wu, K. C., “On the shock enhancement of confined supersonic mixing flows,” Phys. Fluids A, Vol. 3, 1991, pp. 3046-3062.

[11] Marble, F. E., “Gasdynamic enhancement of nonpremixed combustion,” Proc. Combust. Inst., Vol. 25, 1994 , pp. $1-12$.

[12] Brummund, U. and Nuding, J. R.,"Interaction of a compressible shear layer with shock waves - an experimental study," AIAA Paper 97-0392, 1997.

[13] Wepler, U., Huhn, Ch., Koschel, W., "Numerical Simulation of Shock Wave Interaction Effects on Supersonic Mixing Layer Growth,” Numerical Flow Simulation II, Vol. 75, 2001, pp. 217-228.

[14] Zhang, Y., Wang, B., Zhang, H., and Xue, S., "Mixing Enhancement of Compressible Planar Mixing Layer Impinged by Oblique Shock Waves," J. Prop. Power, Vol. 31, No. 1, 2015, pp. 156-169

[15] Seiner, J. M., Dash, S. M., and Kenzakowski, D. C. "Historical Survey on Enhanced Mixing in Scramjet Engines," J. Prop. Power, Vol. 17, No. 6, 2001, pp. 1273-1286.

33 of 35 
[16] Kim, J., Yoon, Y., Jeung, I., Huh, H., and Choi, J., "Numerical Study of Mixing Enhancement by Shock Waves in Model Scramjet Engine,” AIAA Journal, Vol. 41, No. 6, 2003, pp. 1074-1080.

[17] Génin F. and Menon S.,"Studies of shock/turbulent shear layer interaction using Large-Eddy Simulation,” Computers and Fluids, Vol. 39, 5, 2010, pp. 800-819

[18] Kevlahan, N. K-R.,"The propagation of weak shocks in non-uniform flows,” J. Fluid Mech., Vol. 327, 1996, pp. $161-197$.

[19] Lighthill, M. J., "Reflection at a laminar boundary layer of a weak steady disturbance to a supersonic stream, neglecting viscosity and heat conduction," Quarter. J. Mech. Appl. Math., Vol. 3, 1950, pp. 303-325.

[20] Lighthill, M. J., “On boundary layers and upstream influence. II. Supersonic flows without separation,” Proc. Royal Soc. London. Series A., Vol. 213, 1953, pp. 478-507.

[21] Stewartson, K. and Williams, P. G.,"Self-induced separation,” Proc. Royal Soc. London. Series A., Vol. 312, 1969, pp. 181-206.

[22] Nayfeh, A. H.,“Triple-deck structure”, Computers Fluids, Vol. 20, 1991, pp. 269-292.

[23] Riley, N., "Interaction of a shock wave with a mixing region," J. Fluid Mech., Vol. 7, 1960, pp. 321-339.

[24] Moeckel, W. E., "Interaction of oblique shock waves with regions of variable pressure, entropy, and energy," NACA Tech. Note 2725, 1952, pp. 35.

[25] Buttsworth, D. R., "Interaction of oblique shock waves and planar mixing regions," J. Fluid Mech., Vol. 306, 1996, pp. 43-57.

[26] Dolvin, D.,"Hypersonic International Flight Research and Experimentation (HiFiRE)," AIAA Paper 2008-2581, 1985.

[27] Muppala, S. P., Aluri, N. K., Dinkelacker, F. and Leipertz, A., "Development of an algebraic reaction rate closure for the numerical calculation of turbulent premixed methane, ethylene, and propane/air flames for pressures up to 1.0 MPa," Combust. Flame, Vol. 140.4, 2005, pp. 257-266.

[28] Sánchez, A. L. and Williams, F. A., "Recent advances in understanding of flammability characteristics of hydrogen,” Prog. Energy Combust. Sci., Vol. 41, 2014, pp.1-55.

34 of 35 
[29] Rosner, D. E., Transport Processes in Chemically Reacting Flow Systems, Courier Corporation, 2012.

[30] Fernández-Tarrazo, E., Sánchez, A. L. and Williams F. A.,"Hydrogen-air mixing-layer ignition at temperatures below crossover," Combust. Flame, Vol. 160, 2013, pp. 1981-1989.

[31] Lin, C. C., "On the stability of the laminar mixing region between two parallel streams in a gas," NACA Technical Note 2887, 1953.

[32] Papamoschou, P. and Roshko, A., "The compressible turbulent shear layer: an experimental study," J. Fluid Mech., Vol. 197, 1988, pp. 453-477.

[33] Langer, R. E.,"On the asymptotic solutions of differential equations, with an application to the Bessel functions of large complex order," Transact. Amer. Math. Soc., Vol. 34, 1932, pp. 447-487.

[34] Green, J. E., "Interactions between shock waves and turbulent boundary layers," Prog. Aerosp. Sci., Vol.11, 1970 , pp. 235-340.

[35] Stalker, R. J., "Sweepback effects in turbulent boundary-layer shock-wave interaction," J. Aero. Sci., Vol. 27, 1960, pp. 348-356.

[36] Delery, J. M., "Experimental investigation of turbulence properties in transonic shock boundary-layer interactions," AIAA J., Vol. 21, 1983, pp. 180-185.

[37] Delery, J. M., "Shock wave/turbulent boundary layer interaction and its control," Prog. Aerosp. Sci., Vol. 22, 1985, pp. 209-280.

[38] Zel'dovich, Ya. B. and Raizer, Yu. P., Physics of Shock Waves and High-Temperature Hydrodynamic Phenomena, Courier Corporation, 2002. 